\title{
TOPOLOGICAL PROPERTIES OF THE ALGEBRAIC CYCLES FUNCTOR
}

(SUMMER SCHOOL, GRENOBLE 2001)

\author{
PAULO LIMA-FILHO
}

CONTENTS

1. Introduction 2

2. Topological properties of algebraic cycles 4

2.1. The flat and equidimensional topologies $\quad 6$

2.2. The Chow topology 8

2.3. On group completions 10

3. Lawson homology 12

3.1. Basic properties 13

3.2. The homotopy property 13

4. First applications 17

4.1. The $s$-map and cycle maps 17

4.2. Friedlander-Mazur filtrations on cycles 20

5. (Colimits of) Mixed Hodge structures on Lawson homology 21

5.1. A Brief Review of Mixed Hodge Structures 22

5.2. Colimits of M.H.S. and Lawson Homology 23

5.3. A note on the Abel-Jacobi map 28

6. Intersection theory 29

6.1. Deformation to the Normal Cone and Gysin Homomorphism 30

6.2. Intersection Product in Lawson Homology 34

7. Morphic Cohomology 35

7.1. Basic Properties 35

$\begin{array}{lll}\text { 7.2. Duality } & 38\end{array}$

$\begin{array}{ll}\text { References } & 39\end{array}$ 


\section{INTRODUCTION}

In this survey, we present various applications of algebraic cycles, considered as a topological group functor, to the study of complex algebraic varieties. In the classical literature in algebraic geometry, one can already find a vast number of results and techniques that can be considered as precursors of the approach presented here. For example, the study of divisors on Riemann surfaces, Picard groups and Albanese varieties [GH78], Abel-Jacobi maps and generalized Jacobians [Gri68], [Kin83], all contain manifestations of many phenomena that occur when one studies algebraic cycles varying continuously on a family. In the theory presented here, one combines many of these classical ideas with homotopy theory techniques to create the appropriate framework for our study.

The approach used here was introduced in the pioneering work of H. B. Lawson [Law89], whose main objective was to prove his complex suspension theorem(see Theorem 3.11) and, in particular, to compute the homotopy type of algebraic cycles on projective spaces. However, the techniques and ideas introduced in this paper went far beyond these results. In fact, the "complex suspension theorem" itself was a disguised form of a homotopy invariance (cf. Section 3.2). It was due to the insight of E. Friedlander [Fri91] that the techniques used in [Law89] were shown to yield highly non-trivial homology-like functors on projective varieties.

The basic premise is rather simple. Given an algebraic variety $X$, the group $z_{p}(X)$ of algebraic $p$-cycles on $X$ is the free abelian group generated by the irreducible $p$-dimensional subvarieties of $X$. The starting point is the fact that one can endow $z_{p}(X)$ with a well-behaved topology, in a functorial fashion, and hence one can derive various invariants for the variety $X$ in terms of homotopy invariants for $z_{p}(X)$. The primary invariants obtained in this way are called the Lawson homology and the morphic cohomology of $X$, respectively.

We do not follow the historical development of the subject. Instead, we try to present the theory in a more structured way, providing a natural perspective on some of its fundamental features. We hope that this perspective, along with the various exercises included in the text, will have a sound pedagogical value for the non-specialists. In a few places we provide completely new and simpler proofs of key results, and prove generalizations of many others. However, we emphasize that this is not an extensive survey, but rather an incursion in various facets of the theory. For a more comprehensive historical account of the subject, we refer the reader to the excellent survey [Law95].

We compiled result from various sources, notably the works of H. B. Lawson [Law89]; H. B. Lawson and M.-L. Michelsohn [LM88]; E. Friedlander [Fri91], [Fri95]; E. Friedlander and B. Mazur, [FM94]; E. Friedlander and H. B. Lawson, [FL92], [FL98], [FL97]; E. Friedlander and O. Gabber [FG93]; P. Lima-Filho [LF92], [LF93b], [LF94], [LF93a]; H. B. Lawson, P. Lima-Filho 
and M.-L. Michelsohn [LLFM98], [LLFM96], [LLFM]; P. dos Santos [dS]; P. Lima-Filho and P. dos Santos $[\mathrm{dSLF}]$. This is not a complete list, and the author hopes that any unintentional omission will be forgiven.

Each individual section of the survey contains an introduction to its contents, hence we will only give a brief outline of the material here, leaving most citations to the main text.

We start Section 2 with a presentation of three approaches to introduce a topology on $z_{p}(X)$, each one having its own special features. Historically, the first approach used Chow varieties [Law89], [Fri91] on projective varieties, and was subsequently extended to quasiprojective varieties in [LF92]. However, we first introduce topologies using suitable families of cycles, in an approach closer to subsequent works [FL92], [LF94]. In many ways, this goes back to the aforementioned techniques in classical algebraic geometry. Ultimately, these three approaches are shown to coincide. Among their main properties one shows that the assignment $X \mapsto Z_{p}(X)$ is covariantly functorial for proper maps; contravariantly functorial for flat map and transforms closed inclusions into principal fibrations.

In the case where $X$ is projective, the Chow monoid $\mathcal{C}_{p}(X)$ of effective $p$-cycles can be written as a disjoint union of Chow varieties, thus becoming an abelian topological monoid. The inclusion $\mathfrak{C}_{p}(X) \hookrightarrow Z_{p}(X)$ is the universal (näive) group-completion of $\mathfrak{e}_{p}(X)$ in the category of topological monoids. It is certainly desirable that this group-completion, in the level of spaces, also have suitable homotopy theoretic properties. We conclude the section with a discussion of the homotopy theoretic properties of $z_{p}(X)$, in projective case. This is done by exhibiting a simplicial space, a triple bar construction $B\left(\mathcal{C}_{p}(X) \times \mathcal{C}_{p}(X), \mathfrak{C}_{p}(X)\right.$, *), whose geometric realization is homotopy equivalent to the homotopy group completion $\Omega B \mathcal{C}_{p}(X)$. It turns out that $z_{p}(X)$ is also homotopy equivalent to the geometric realization of this simplicial space, and hence $z_{p}(X) \cong \Omega B \mathcal{C}_{p}(X)$. We describe this simplicial variety in detail, for we will use this model subsequently, when we discuss mixed Hodge structures in Section 5.

In Section 3 we introduce the Lawson homology $L_{*} H_{*}(X)$ of a complex variety $X$, a bigraded group where $L_{p} H_{n}(X)$ is defined as the homotopy groups $\pi_{n-2 p}\left(Z_{p}(X)\right)$. The topological properties of $z_{p}(X)$ described in Section 2 immediately yield the functorial properties of Lawson homology, including localization sequences. We end the section by discussing Lawson's suspension theorem and its main consequence, the homotopy property, stating that the flat pull-back map $p^{*}: z_{p}(X) \rightarrow z_{p+e}(E)$ induced by a vector bundle projection $p: E \rightarrow X$ is a homotopy equivalence, inducing, in particular, an isomorphism in Lawson homology.

The first applications of the properties above are described in Section 4, where we present Friedlander-Mazur's [FM94] $s$-map $s: L_{p} H_{n}(X) \rightarrow L_{p-1} H_{n}(X)$ and its associated cycle map $s^{p}: L_{p} H_{n}(X) \rightarrow H_{n}(X ; \mathbb{Z})$ from Lawson homology to the Borel-Moore homology of $X$. We extend the definition of the $s$-map to arbitrary varieties and provide a novel presentation of the map 
and its functoriality. One of the most interesting applications of this construction is FriedlanderMazur's filtration on the Griffiths groups, interpolating between the filtrations introduced by Nori in [Nor93] and Bloch-Ogus in [BO84].

In Section 5 we use the simplicial space model for $z_{p}(X)$ described in $\S 2.3$ to provide a structure of colimits of mixed Hodge structures on the homology of cycle spaces $z_{p}(X)$, for arbitrary complex varieties $X$. We show that this structure coincides with Friedlander-Mazur's in the case where $X$ is projective. Using the Hurewicz map we also endow Lawson homology with a colimit of mixed Hodge structures.

The intersection theory for Lawson homology, developed by Friedlander and Gabber [FG93], is explained in 6. Here we work directly on the homotopy category, instead of the original derived category approach, transforming the deformation to the normal cone technique into a homotopy lifting-extension problem. This gives Gysin (homotopy class of) maps $j^{!}: z_{p}(X) \rightarrow z_{p-e}$ associated to regular embeddings $j: Y \hookrightarrow X$ of codimension $e$. The intersection product for the Lawson homology of smooth varieties is then defined in the standard way using the Gysin map associated to the diagonal embedding $X \hookrightarrow X \times X$.

In the last section we briefly present the cohomological counterpart of Lawson homology, the morphic cohomology group $L^{*} H^{*}(X)$, introduced by E. Friedlander and H. B. Lawson in [FL92]. A more thorough description of its properties and the body of work associated to morphic cohomology would require an equally lengthy survey, beyond the scope of this one. We simply present the natural relation between morphic and ordinary cohomology, as a motivation to its definition. Then we conclude displaying the surprisingly natural duality map from morphic cohomology to Lawson homology. In the deep and beautiful papers [FL98] and [FL97], it is shown, among many other results that the duality map gives an isomorphism when the varieties are smooth.

ACKNOWLEDGEMENTS: The author would like to thank the hospitality of the Institut Fourier during the Summer School 2001, and to thank the organizers Chris Peters and Stefan MüllerStach, for the opportunity to participate in such an stimulating event and for their infinite patience for my tardiness in preparing these notes.

\section{TOPOLOGICAL PROPERTIES OF ALGEBRAIC CYCLES}

Throughout these lectures an algebraic variety is a reduced scheme of finite type over $\mathbb{C}$. To avoid excessive notation, we sometimes use the same letter $X$ to denote either the variety $X$ or the space of complex points $X(\mathbb{C})$ of $X$ with the analytic topology. We hope that the context will suffice to determine the meaning of the notation used. 
Our goal here is to describe a topology on the group $z_{p}(X)$ of algebraic $p$-cycles on an arbitrary complex variety $X$. We present this topology in detail below and prove some the following properties. These properties are a compilation of results proven in [Law89], [Fri91], [LF92] and [LF94].

Property 1: $z_{p}(X)$ is a Hausdorff topological group of the homotopy type of a $C W$ complex.

Property 2: The connected component $z_{p}(X)_{o}$ of the identity element $0 \in z_{p}(X)$ is the group $z_{p}(X)$ alg of $p$-cycles algebraically equivalent to zero.

Property 3: If $f: X \rightarrow Y$ is a proper morphism, then the push-forward map $f_{*}: z_{p}(X) \rightarrow$ $z_{p}(Y)$ is a continuous homomorphism. If $f: X \rightarrow Y$ is a flat morphism of relative dimension $k$, then the flat pull-back $f^{*}: z_{p}(Y) \rightarrow z_{p+k}(X)$ is a continuous homomorphism.

Property 4: If $Y \subseteq X$ is closed subvariety, then $z_{p}(Y) \hookrightarrow z_{p}(X)$ is a closed embedding and the sequence $z_{p}(Y) \hookrightarrow z_{p}(X) \rightarrow z_{p}(X-Y)$ is a principal fibration.

Remark 2.1. The fibration described in Property 4 is functorial with respect to maps of pairs satisfying the conditions of Property 3.

The following additional properties are instrumental in many situations.

Property 5: The topology on $z_{p}(X)$ is given as the direct limit topology of a filtering sequence

$$
z_{p}(X)_{\leq 1} \subseteq z_{p}(X)_{\leq 2} \subseteq \cdots \subseteq z_{p}(X)_{\leq d} \subseteq \cdots
$$

of closed, compact subsets of $z_{p}(X)$, satisfying:

(1) The inclusions $z_{p}(X)_{\leq d} \subseteq z_{p}(X)_{\leq d+1}$ are closed cofibrations;

(2) If $X$ is quasiprojective, the successive differences $z_{p}(X)_{\leq d+1}-z_{p}(X)_{\leq d}$ are homeomorphic to quasiprojective varieties;

(3) The filtration is compatible with the group operation.In other words,

$$
z_{p}(X)_{\leq d}+z_{p}(X)_{\leq e} \subseteq z_{p}(X)_{\leq d+e}
$$

We will present three different approaches to introduce such a topology on $z_{p}(X)$, each one having its own merits and natural properties. At the end, one can show that the three approaches produce the same topology; cf. [LF94]. 
2.1. The flat and equidimensional topologies. The first two approaches are introduced à la Bourbaki, using algebraic families of cycles.

Definition 2.2. Let $\mathcal{F}=\left\{i_{\lambda}: S_{\lambda} \rightarrow T\right\}_{\lambda \in \Lambda}$ be a family of maps from topological spaces $S_{\lambda}$ into a set $T$. The collection $\tau_{\mathcal{F}}$ of subsets $U \subset T$ such that $i_{\lambda}^{-1}(U)$ is open in $S_{\lambda}$, for all $\lambda \in \Lambda$, defines a topology on $T$, the finest topology making all the maps $i_{\lambda}$ continuous. Denote by $T^{\mathcal{F}}$ or $\left(T, \tau_{\mathcal{F}}\right)$ the resulting topological space.

Remark 2.3. Given $\left(T, \tau_{\mathcal{F}}\right)$ as above, one can show that a map $f: T \rightarrow Y$ from $T$ to a space $Y$ is continuous if and only if for each $i_{\lambda}: S_{\lambda} \rightarrow T$ in $\mathcal{F}$ the composition $f \circ i_{\lambda}: S_{\lambda} \rightarrow Y$ is continuous.

Our two primary examples are the following.

Example 2.4 (Flat FAMILIES). Consider an algebraic variety $X$, and let $(S, \sigma)$ be a pair consisting of an algebraic variety $S$ of pure dimension $k$, and an algebraic cycle $\sigma=\sum_{i} n_{i} \Gamma^{i}$ on $S \times X$ which is flat of relative dimension $p$ over $S$. One can define a map $\pi_{\sigma}: S(\mathbb{C}) \rightarrow Z_{p}(X)$ defined on a closed point $s \in S(\mathbb{C})$ by $\pi_{\sigma}(s)=\sum_{i} n_{i}\left[\Gamma_{s}^{i}\right]$, where $\left[\Gamma_{s}^{i}\right]$ is the cycle associated to the scheme-theoretic fiber $\Gamma_{s}^{i}$; cf. [Ful84, $\left.\S 1.5\right]$.

Let $z_{p}(X / S)^{\mathrm{fl}}$ denote the group of all cycles in $S \times X$ which are flat of relative dimension $p$ over $S$, and define

$$
\mathcal{F}_{p}(X)^{\mathrm{fl}}:=\left\{\pi_{\sigma}: S(\mathbb{C}) \rightarrow \mathcal{Z}_{p}(X) \mid \sigma \in \mathcal{Z}_{p}(X / S)^{\mathrm{fl}} \text { and } S \text { is pure dimensional. }\right\}
$$

If one gives $S(\mathbb{C})$ the analytic topology, then $\mathcal{F}_{p}(X)^{\mathrm{fl}}$ becomes a family of maps from a collection of topological spaces to the set $z_{p}(X)$. Using Definition 2.2 one obtains a unique flat topology on $z_{p}(X)$, which we denote by $z_{p}(X)^{\mathrm{fl}}$.

In a similar fashion, we can replace flat families over arbitrary base spaces by equidimensional families over smooth base spaces.

Example 2.5 (EQUIDIMENSIONAL FAMILIES). Let $z_{p}(X / S)^{\mathrm{e} q}$ denote the group of all cycles $\sigma$ in $S \times X$ which are equidimensional of relative dimension $p$ over a smooth base $S$. For a closed point $s \in S(\mathbb{C})$ define $\pi_{\sigma}(s)=\sum_{i} n_{i}\left[\Gamma_{s}^{i}\right] \in \mathcal{Z}_{p}(X)$, where $\left[\Gamma_{s}^{i}\right]$ is the intersection-theoretic fiber $\Gamma^{i} \cdot(s \times X)$ over $s$. Define

$$
\mathcal{F}_{p}(X)^{\mathrm{eq}}:=\left\{\pi_{\sigma}: S(\mathbb{C}) \rightarrow z_{p}(X) \mid \sigma \in \mathcal{Z}_{p}(X / S)^{\mathrm{e} q} \text { and } S \text { is smooth. }\right\} .
$$

If one gives $S(\mathbb{C})$ the analytic topology, then $\mathcal{F}_{p}(X)^{\mathrm{fl}}$ becomes a family of maps from a collection of topological spaces to the set $z_{p}(X)$. Using Definition 2.2 one obtains a unique equidimensional topology on $z_{p}(X)$, which we denote by $z_{p}(X)^{\mathrm{fl}}$. 
Exercise 2.1. (1) Prove that, given an algebraic variety $X$, the flat topology is completely determined by the subfamily

$$
\mathcal{F}_{p}(X)_{\text {smooth }}^{\mathrm{fl}}:=\left\{\pi_{\sigma}: S(\mathbb{C}) \rightarrow \mathcal{Z}_{p}(X) \mid \sigma \in \mathcal{Z}_{p}(X / S)^{\mathrm{fl}} \text { and } S \text { is smooth. }\right\} \subset \mathcal{F}_{p}(X)^{\mathrm{fl}} .
$$

(2) Let $f: X \rightarrow Y$ be a flat morphism of relative dimension $k$. Prove that the flat pull-back $f^{*}: z_{p}(Y) \rightarrow z_{p+k}(Y)$ is a continuous map.

The behavior of these topologies under proper push-forward is a bit more subtle. Consider a proper map $f: X \rightarrow Y$, and let $\Gamma \subset S \times X$ be an irreducible subvariety, flat over a smooth variety $S$. Define $\Gamma^{\prime}=(1 \times f)(\Gamma) \subset S \times Y$. The projection $\Gamma \rightarrow S$ factors as a composition $\Gamma \stackrel{p}{\rightarrow} \Gamma^{\prime} \stackrel{g}{\rightarrow} S$, where $p$ is surjective and $g \circ p$ is flat. In particular, $g \circ p$ is universally open, and so is $g$; cf. [GD66, Prop. 14.3.4(i)]. On the other hand, since $S$ is normal and $\Gamma^{\prime}$ is irreducible, these conditions show that $\Gamma^{\prime} \rightarrow S$ is equidimensional over $S$; cf. [GD66, Corollary 14.4.9(b)]. Now, given $s \in S(\mathbb{C})$, it follows from [Ful84, Thm. 6.2(a)] that

$$
\pi_{\Gamma^{\prime}}(s):=\pi_{(1 \times f)(\Gamma)}(s)=f_{*}\left(\pi_{\Gamma}(s)\right),
$$

where the map $\pi_{\Gamma^{\prime}}$ is defined as in Example 2.5 and $\pi_{\Gamma}$ is defined in Example 2.4. This observation, along with Remark 2.3, proves the following.

Lemma 2.6. Give a proper morphism $f: X \rightarrow Y$, the push-forward homomorphism $f_{*}$ : $z_{p}(X)^{f l} \rightarrow z_{p}(Y)^{e q}$ is a continuous map.

This lemma provides the first step in comparing the two topologies. The next step, uses the following flattification/extension result.

Theorem 2.7. Let $j: U \hookrightarrow V$ be a dense open subvariety of an algebraic variety $V$, and let $S$ be a smooth $k$-dimensional affine variety. Given a subvariety $\Gamma \subset S \times U$, which is equidimensional of relative dimension p over $S$, there is a blow-up $b: \tilde{S} \rightarrow S$ and a subvariety $\tilde{\Gamma}$ of $\tilde{S} \times V$ flat over $\tilde{S}$, with relative dimension $P$ and satisfying:

a: $\tilde{\Gamma}$ is the proper transform of the closure $\bar{\Gamma}$ of $\Gamma$ in $S \times V$;

b: The diagram

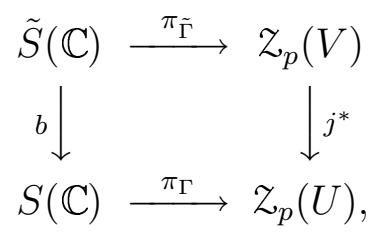

commutes, where $\pi_{\tilde{\Gamma}} \in \mathcal{F}_{p}(X)^{f l}$ and $\pi_{\Gamma} \in \mathcal{F}_{p}(X)^{e q}$.

The next result follows from this proposition.

Theorem 2.8. [LF94, Cor. 3.5] Let $j: U \hookrightarrow V$ be the inclusion of open dense subvariety of $V$. Then the restriction homomorphism $j^{*}: z_{p}(V)^{f l} \rightarrow z_{p}(U)^{e q}$ is a quotient map. 
Corollary 2.9. Given an algebraic variety $X$, the identity map induces a homeomorphism $i_{*}$ : $z_{p}(X)^{f l} \rightarrow z_{p}(X)^{e q}$.

Proof. It follows from the Theorem that the identity $i: X \rightarrow X$ induces a closed map $i_{*}$ : $z_{p}(X)^{\mathrm{fl}} \rightarrow z_{p}(X)^{\mathrm{eq}}$, and Lemma 2.6 shows that this map is continuous.

Corollary 2.10. Proper push-forwards and flat pull-backs are continuous maps in the flat (=equidimensional) topology on groups of algebraic cycles.

2.2. The Chow topology. One can introduce yet a third topology on $z_{p}(X)$, the Chow topology $z_{p}(X)^{\text {ch }}$. This approach allows one to prove various point-set topological properties of $z_{p}(X)$. The definition, however, requires a few more steps than the flat and equidimensional topologies.

\section{Case 1: Projective varieties.}

Given a projective embedding $X \subseteq \mathbb{P}^{n}$, let

$$
\mathcal{C}_{p}(X):=\amalg_{\alpha \in \Pi_{p}(X)} \mathfrak{e}_{p, \alpha}(X)
$$

denote the Chow monoid of $X$. This is the free abelian monoid generated by the irreducible $p$-dimensional subvarieties of $X$. One can write $\mathcal{C}_{p}(X)$ as a disjoint union of Chow varieties $\mathcal{e}_{p, \alpha}(X)$. These are connected projective varieties, indexed by the monoid $\Pi_{p}(X)$ of effective algebraic equivalence classes of effective algebraic p-cycles. There is a monoid morphism deg : $\Pi_{p}(X) \rightarrow \mathbb{Z}_{\geq 0}$ such that $\operatorname{deg}^{-1}(d)$ is finite and

$$
\mathcal{C}_{p, d}(X):=\amalg_{\alpha \in \operatorname{deg}-1(d)} \mathcal{C}_{p, \alpha}(X)
$$

is a finite union of closed connected subvarieties of the classical Chow variety $\mathcal{C}_{p, d}\left(\mathbb{P}^{n}\right)$ of effective cycles of degree $d$ in $\mathbb{P}^{n}$.

Remark 2.11. The addition map $+: \mathcal{C}_{p}(X) \times \mathcal{C}_{p}(X) \rightarrow \mathcal{C}_{p}(X)$ induces algebraic maps + : $\mathcal{C}_{p, d}(X) \times \mathcal{C}_{p, e}(X) \rightarrow \mathcal{C}_{p, d+e}(X)$.

The group $z_{p}(X)$ is the Grothendieck group of the monoid $\mathcal{e}_{p}(X)$, which can be described as the quotient of $\mathcal{C}_{p}(X) \times \mathcal{C}_{p}(X)$ under the relation $(a, b) \sim\left(a^{\prime}, b^{\prime}\right)$ if and only if $a+b^{\prime}=a^{\prime}+b$. Now, define $Z_{p}(X)^{\mathrm{ch}}$ as the quotient topology induced by the quotient map $\rho: \mathcal{C}_{p}(X) \times \mathcal{C}_{p}(X) \rightarrow$ $z_{p}(X)$.

Several properties follow from this definition. If one defines

$$
z_{p}(X)_{\leq d}:=\rho\left(\amalg_{r+s \leq d} \mathcal{e}_{p, r}(X) \times \mathcal{C}_{p, s}(X)\right) \subset z_{p}(X),
$$

then one can show that the topology on $z_{p}(X)$ is the direct limit topology induced by this filtration, as described in Property 5. In fact, in this context we can prove Properties 1, 3 and 5 for the Chow topology, in the case of projective varieties, by using basic results from [Ste67], 
and certain cofibration properties resulting from triangulations of complex algebraic varieties; cf. [Hir75]

\section{Case 2: Quasiprojective varieties.}

Given a closed subvariety $Y$ of a projective variety $X$, it is easy to see that show that $z_{p}(Y)^{\text {ch }}$ is a closed subgroup of $z_{p}(X)^{\text {ch }}$; cf. [LF92]. The following result allows one to extend the Chow topology to arbitrary varieties, and has many deep consequences.

Theorem 2.12 ([LF92]). If $f:(X, Y) \rightarrow\left(X^{\prime}, Y^{\prime}\right)$ is a relative isomorphism of pairs of projective varieties, then it induces a topological group isomorphism

$$
f_{*}: Z_{p}(X)^{c h} / z_{p}(Y)^{c h} \rightarrow Z_{p}\left(X^{\prime}\right)^{c h} / Z_{p}\left(Y^{\prime}\right)^{c h} .
$$

With this result, one shows that the following definition is independent of projective embeddings and compactifications.

Definition 2.13 ([LF92]). Let $U \subset \mathbb{P}^{n}$ be a quasiprojective variety. Define $z_{p}(U)^{\text {ch }}$ as the topological group quotient $z_{p}(\bar{U})^{\mathrm{ch}} / z_{p}(\bar{U}-U)^{\mathrm{ch}}$.

\section{Case 3: Arbitrary varieties.}

An envelope of a variety $X$ is a proper morphism $p: X^{\prime} \rightarrow X$ such that for every closed irreducible subvariety $V$ of $X$ there is a subvariety $V^{\prime}$ of $X^{\prime}$ such that $p$ maps $V^{\prime}$ birrationally onto $V$. We call $p$ a Chow envelope if, in addition, $X^{\prime}$ is a quasiprojective variety. One can show that any variety of finite type $X$ has a Chow envelope, and that envelopes are preserved under base-extension; cf. [FG83]. Observe that if $p: X^{\prime} \rightarrow X$ is an envelope, then the induced (proper push-forward) homomorphism $p_{*}: z_{p}\left(X^{\prime}\right) \rightarrow z_{p}(X)$ is surjective.

Definition 2.14. Given a variety $X$ and a Chow envelope $p: X^{\prime} \rightarrow X$, define the Chow topology $z_{p}(X)^{\text {ch }}$ to be the quotient topology on $z_{p}(X)$ induced by the surjection $p_{*}: z_{p}\left(X^{\prime}\right)^{\text {ch }} \rightarrow z_{p}(X)$. It is easy to see that this is independent of the Chow envelope.

Remark 2.15. In this case one defines $z_{p}(X)_{\leq d}=p_{*}\left(j^{*} z_{p}\left(\overline{X^{\prime}}\right)_{\leq d}\right)$, where $j: X \hookrightarrow \overline{X^{\prime}}$ is a projective compactification of $X^{\prime}$. This filtration expresses the topology on $Z_{p}(X)$ as a colimit of compact subsets, and is the basis of various inductive arguments used in proving Properties 1, 3 and 5.

It is easy to compare the Chow and flat topologies in the case of a projective variety $X \subset \mathbb{P}^{n}$, for one can use finitely many Hilbert schemes $H i l b_{X}^{P}$ parametrizing subschemes of $\mathbb{P}^{n}$, whose Hilbert polynomial has leading coefficients $\frac{d}{p !} t^{p}$, to produce a surjective proper map

$$
\rho_{d}: \amalg_{P} H i l b_{X}^{P} \rightarrow \mathcal{C}_{p, d}(X)
$$

for all $d$. These maps, along with the universal flat families over Hilbert schemes, provide the desired comparison. 
Theorem 2.16. If $X$ is a projective variety then the identity map induces a homeomorphism $i: z_{p}(X)^{f l} \rightarrow z_{p}(X)^{c h}$.

Corollary 2.17. The conclusion of the theorem still holds for quasiprojective varieties.

Proof. Let $j: U \hookrightarrow \bar{U} \subset \mathbb{P}^{n}$ be a projective compactification of $U$. One has a commutative diagram

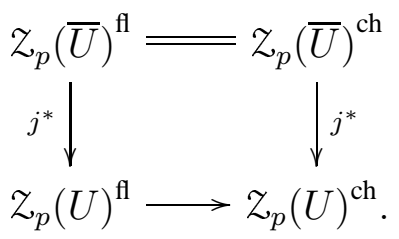

where the left vertical arrow is a quotient map, according to Theorem 2.8, and the right vertical arrow is a quotient map by definition. This suffices to prove the corollary.

Remark 2.18. The result holds for arbitrary varieties, and the proof follows from the same flattification/extension result as Theorem 2.8 does. Roughly speaking, one just needs to show that any flat family of cycles in $X$ can be lifted to a Chow envelope $p: X^{\prime} \rightarrow X$ after a proper base change.

Once we have proven equality of all three topologies, we simply denote the resulting topological group by $z_{p}(X)$.

\subsubsection{Exercises.}

Exercise 2.2. Prove the following assertions.

(1) Given an algebraic variety $X$, the flat topology is completely determined by the subfamily $\mathcal{F}_{p}(X)_{\text {smooth }}^{\mathrm{fl}}:=\left\{\pi_{\sigma}: S(\mathbb{C}) \rightarrow \mathcal{Z}_{p}(X) \mid \sigma \in \mathcal{Z}_{p}(X / S)^{\mathrm{fl}}\right.$ and $S$ is smooth. $\} \subset \mathcal{F}_{p}(X)^{\mathrm{fl}}$.

(2) Let $f: X \rightarrow Y$ be a flat morphism of relative dimension $k$. Prove that the flat pull-back $f^{*}: z_{p}(Y) \rightarrow z_{p+k}(Y)$ is a continuous map.

2.3. On group completions. In this section we make a brief discussion of homotopy theoretic properties of the group $z_{p}(X)$ when $X$ is projective. A more extensive study, for a broader class of monoids, can be found in [LF93a].

Consider an arbitrary topological monoid $M$. If $(A, M, B)$ is a triple where $A$ is a right $M$ space and $B$ is a left $M$-space, then one can construct a simplicial space $\mathcal{B}_{*}(A, M, B)$, called the triple bar construction, as follows; cf. [May75].

The space of $n$-simplices is defined as

$$
\mathcal{B}_{n}(A, M, B):=A \times M^{\times n} \times B ;
$$


with faces defined by

$$
d_{i}\left(a ; m_{1}, \ldots, m_{n} ; b\right):= \begin{cases}\left(a * m_{1} ; m_{2}, \ldots, m_{n} ; b\right) & ; \text { for } i=0 \\ \left(a ; m_{1}, \ldots, m_{i} m_{i+1}, \ldots, m_{n} ; b\right) & ; \text { for } 1 \leq i<n \\ \left(a ; m_{1}, \ldots, m_{n-1} ; m_{n} * b\right) & ; \text { for } i=n\end{cases}
$$

and degeneracies $s_{i}\left(a ; m_{1}, \ldots, m_{n} ; b\right)=\left(a ; m_{1}, \ldots, m_{i-1}, 0, m_{i}, \ldots, m_{n} ; b\right)$, where 0 is the identity element of the monoid.

This construction is functorial on triples and its geometric realization $\mathcal{B}(A, M, B)$ satisfies the following properties. We refer to reader to [May75] for details on this construction.

(1) If $(A, M, B)$ is such a triple and $M$ acts trivially on $C$, then $\mathcal{B}(C \times A, M, B)=C \times$ $\mathcal{B}(A, M, B)$, where $M$ acts diagonally on $C \times A$; cf. [May75].

(2) $\mathcal{B}(*, M, *)=B M$ is the classifying space of $M$ and the map $E M:=\mathcal{B}(M, M, *) \rightarrow$ $B M$ induced by the obvious map of triples $(M, M, *) \rightarrow(*, M, *)$ is the universal quasifibration for $M$; cf. [May75].

(3) Given a triple of the form $(A, M, *)$, the bar construction $\mathcal{B}(A, M, *)$ is the homotopy quotient of $A$ under the action of $M$;

(4) If $M$ is abelian, then $B M$ is an abelian monoid and so is $\Omega B M$ under pointwise addition.

The following result seems to be well-known, according to the observation [Seg74, p. 305], but the first proof known to the author is found in [LF93a].

Proposition 2.19. If $M$ is abelian, and $(M \times M, M, *)$ is the triple where $M$ acts diagonally on $M \times M$, then $\Omega B M$ is naturally homotopy equivalent to $\mathcal{B}(M \times M, M, *)$, which is the homotopy quotient of $M \times M$ by the diagonal action.

Remark 2.20. (1) In [Seg74, p. 305], Segal mentions that the result still holds if the monoid is "sufficiently abelian", by which we presume that this means up to sufficiently high coherent homotopies. We only present the proof in the case where $M$ is actually abelian.

(2) The involution $M \times M \rightarrow M \times M$ sending $(m, n)$ to $(n, m)$ induces an involution $\iota_{M}: \mathcal{B}(M \times M, M, *) \rightarrow \mathcal{B}(M \times M, M, *)$ which is natural on $M$ and corresponds to giving the "inverse" of an element. In other words, $i d+\iota_{M}$ is naturally homotopic to zero.

The Proposition allows one to use the model $\mathcal{B}(M \times M, M, *)$ for the homotopy theoretic group-completion $\Omega B M$ of an abelian monoid $M$. Note that one has a map of triples $(M \times$ $M, M, *) \rightarrow\left(M^{+}, *, *\right)$, where $M^{+}$is the Grothendieck group of the monoid, with the quotient topology from $M \times M$. It turns out that, under mild conditions on the monoid $M$, the induced map on triple bar constructions is a homotopy equivalences, as we explain below. 
Definition 2.21. Let $M$ be a monoid whose topology is given by a filtration $\cdots \subseteq M_{n} \subset M_{n+1} \subset$ $\cdots$. Define $(M \times M)_{d}=\cup_{r+s \leq d}\left(M_{r} \times M_{s}\right) \subset M \times M$, and

$$
\begin{array}{r}
\Delta(M \times M)_{d}:=\left\{\left(m, m^{\prime}\right) \in(M \times M)_{d} \mid\left(m+a, m^{\prime}+a\right)=\left(n+b, n^{\prime}+b\right)\right. \\
\left.\quad \text { for some }\left(n, n^{\prime}\right) \in(M \times M)_{d-1} \text { and } a, b \in M\right\}
\end{array}
$$

Define $M$ to be properly $c$-filtered if $M_{d}$ is compact for all $d$ and the inclusion $\Delta(M \times M)_{d} \subseteq$ $(M \times M)_{d}$ is a cofibration.

Example 2.22. Given a projective variety $X$, every Chow monoid $\mathcal{C}_{p}(X)$ is properly $c$-filtered. Actually, it is $c$-graded in the sense that the filtration comes from a grading.

Theorem 2.23 ([LF93a]). Let $M$ be a properly c-graded abelian topological monoid. Then the map

$$
B(M \times M, M, *) \rightarrow M^{+}
$$

is a homotopy equivalence.

Corollary 2.24. For every projective variety $X$, one has a homotopy equivalence

$$
z_{p}(X) \cong \Omega B\left(\mathcal{C}_{p}(X)\right)
$$

Remark 2.25. The definition above can be extended to actions of $M$ on a space $A$, mimicking the properties of the action of the diagonal on $M \times M$, as defined above. This condition was called a tractable action of $M$ on $A$ in [FG93]. The proof of the theorem yields the fact that under appropriate cofibrant filtration condition, the homotopy quotient $\mathcal{B}(A, M, *)$ is homotopy equivalent to the actual quotient $A / M$ of $A$ by the action of the monoid.

\section{LAWSON HOMOLOGY}

The Lawson homology groups of an algebraic variety $X$ are defined in terms of the homotopy groups of the various topological groups $z_{p}(X)$. They form a family of invariants for the variety $X$ that encodes hybrid properties of $X$, interpolating from purely topological invariants on one end to purely algebraic on the other. The term Lawson homology for projective varieties was coined by E. Friedlander in [Fri91] after Lawson's work [Law89]. In [Fri91] an $\ell$-adic version of Lawson homology is developed for projective varieties over fields of characteristic $p \neq \ell$. Subsequently, the theory was extended to arbitrary (complex) varieties in [LF92] and [LF94].

Definition 3.1. Define the Lawson homology groups of $X$ as

$$
L_{p} H_{n}(X):=\pi_{n-2 p}\left(z_{p}(X)\right),
$$

for $n \geq 2 p$ and $0 \leq p \leq \operatorname{dim} X$. 
3.1. Basic properties. The topological properties of the functors $X \mapsto z_{p}(X)$, described in the previous section, along with basic properties of homotopy groups, yield the following basic properties.

Theorem 3.2. The Lawson homology functor satisfies the following properties.

Proper push-forward: It is a covariant functor for proper morphisms. In other words, a proper morphism $f: X \rightarrow Y$ induces group homomorphisms $f_{*}: L_{p} H_{n}(X) \rightarrow$ $L_{p} H_{n}(Y)$, and $(f \circ g)_{*}=f_{*} \circ g_{*}$ for proper morphisms $f, g$.

Flat pull-back: It is contravariant for flat morphisms. In other words, a flat morphism $f: X \rightarrow Y$ of relative dimension $k$ induces group homomorphisms $f^{*}: L_{p} H_{n}(Y) \rightarrow$ $L_{p+k} H_{n+2 k}(X)$, and $(f \circ g)^{*}=g^{*} \circ f^{*}$ for flat morphisms $f, g$.

Localization sequence: Given a closed subvariety $Y \subset X$ one has a long exact sequence

$$
\cdots \rightarrow L_{p} H_{n+1}(X-Y) \rightarrow L_{p} H_{n}(Y) \rightarrow L_{p} H_{n}(X) \rightarrow L_{p} H_{n}(X-Y) \rightarrow \cdots
$$

In special cases one can recover classical invariants out of Lawson homology:

Theorem 3.3. Let $X$ be an algebraic variety.

a: [Fri91] $L_{p} H_{2 p}(X):=\pi_{0}\left(Z_{p}(X)\right) \cong \mathcal{A}_{p}(X)$, where $\mathcal{A}_{p}(X)$ is the group of algebraic p-cycles on $X$ modulo algebraic equivalence.

b: [DT56] $L_{0} H_{n}(X):=\pi_{n}\left(Z_{0}(X)\right) \cong H_{n}^{B M}(X(\mathbb{C}) ; \mathbb{Z})$, where the latter denotes the BorelMoore homology of the analytic space $X(\mathbb{C})$ with coefficients in $\mathbb{Z}$.

c: [Fri91] Given a non-singular projective variety $X$ of dimension $n$, there are isomorphisms:

$$
\begin{aligned}
L_{n-1} H_{2 n}(X) & \cong \mathbb{Z} \\
L_{n-1} H_{2 n-1}(X) & \cong H_{2 n-1}(X ; \mathbb{Z}) \\
L_{n-1} H_{2 n-2}(X) & \cong N S(X),
\end{aligned}
$$

where the latter is the Neron-Severi group $H_{n-1, n-1}(X ; \mathbb{Z})$.

3.2. The homotopy property. The fundamental result that triggered the development of this theory was B. Lawson's seminal work [Law89]. In order to present his complex suspension theorem $^{1}$, instead of following the historical development of the subject we first introduce the join pairing of cycles, a construction that will appear in multiple occasions heretofore.

\footnotetext{
${ }^{1}$ This was Lawson's original terminology.
} 
3.2.1. The join pairing. Given a finite dimensional complex vector space $V$, let $\mathbb{P}(V)$ denote the projective space of complex lines trough the origin in $V$. In other words, $\mathbb{P}(V):=\operatorname{Proj}\left(\operatorname{Sym}_{*}(\check{V})\right)$, where $\check{V}$ denotes the dual of $V$. Let $Z \subset \mathbb{P}(V)$ and $Z^{\prime} \subset \mathbb{P}(W)$ be irreducible subvarieties of dimensions $r$ and $s$ and degrees $d$ and $e$, respectively. Under the natural embeddings $\mathbb{P}(V) \subset \mathbb{P}(V \oplus W)$ and $\mathbb{P}(W) \subset \mathbb{P}(V \oplus W)$ as two disjoint linear subspaces, one can consider both $Z$ and $Z^{\prime}$ as subvarieties of $\mathbb{P}(V \oplus W)$. Define the algebraic join $Z \# Z^{\prime}$ of $Z$ and $Z^{\prime}$ as the subvariety of $\mathbb{P}(V \oplus W)$ consisting of all projective lines joining points in $Z$ to points in $Z^{\prime}$.

\section{Remark 3.4.}

(1) Observe that if $C Z \subset V$ denotes the affine cone over $Z$ and $C Z^{\prime}$ is the corresponding cone for $Z^{\prime}$ then $Z \# Z^{\prime}$ is the subvariety of $\mathbb{P}(V \oplus W)$ whose affine cone is $C Z \times C Z^{\prime}$. This shows that $Z \# Z^{\prime}$ is indeed an irreducible subvariety of dimension $r+s+1$, where $r=\operatorname{dim} Z$ and $s=\operatorname{dim} S$.

(2) It can also be shown that if $\operatorname{deg} Z=d$ and $\operatorname{deg} Z^{\prime}=e$, then $\operatorname{deg}\left(Z \# Z^{\prime}\right)=d \cdot e$.

Definition 3.5. Let $X$ and $X^{\prime}$ be projective varieties, with respective embeddings $j: X \hookrightarrow \mathbb{P}(V)$ and $j^{\prime}: X^{\prime} \hookrightarrow \mathbb{P}(W)$. The join pairing

$$
\#: z_{r}(X) \times z_{r^{\prime}}(X) \rightarrow z_{r+s}\left(X \# X^{\prime}\right)
$$

is the bilinear extension of the join of subvarieties of $X$ and $X^{\prime}$. That is, for cycles $\sigma=\sum_{i} n_{i} S_{i} \in$ $z_{r}(X)$ and $\tau=\sum_{j} m_{j} T_{j} \in Z_{s}\left(X^{\prime}\right)$, one has $\sigma \# \tau=\sum_{i, j} n_{i} m_{j} S_{i} \# T_{j}$. as described above.

Proposition 3.6. For projective subvarieties $X \subset \mathbb{P}(V)$ and $X^{\prime} \subset \mathbb{P}(W)$, the join pairing $\#: z_{r}(X) \times z_{r^{\prime}}(X) \rightarrow z_{r+s}\left(X \# X^{\prime}\right)$ is a continuous map.

Proof. Since $X, X^{\prime}$ and $X \# X^{\prime}$ are closed subvarieties of their corresponding projective spaces, it follows from Property 4 in Section 2 that one only needs to prove continuity of the pairing \# : $z_{r}(\mathbb{P}(V)) \times z_{r^{\prime}}(\mathbb{P}(W)) \rightarrow z_{r+s+1}(\mathbb{P}(V \oplus W))$. In this case, consider the blow-up $B$ of $\mathbb{P}(V \oplus W)$ along $\mathbb{P}(V) \amalg \mathbb{P}(W)$. It is easy to see that $B=\mathbb{P}\left(p_{1}^{*}\left(\mathcal{O}_{\mathbb{P}(V)}(-1) \oplus p_{2}^{*}\left(\mathcal{O}_{\mathbb{P}(W)}(-1)\right)\right.\right.$, where $p_{1}: \mathbb{P}(V) \times \mathbb{P}(W) \rightarrow \mathbb{P}(V)$ and $p_{2}: \mathbb{P}(V) \times \mathbb{P}(W) \rightarrow \mathbb{P}(V)$ are the respective projections, and $\mathcal{O}(-1)$ denotes tautological bundles. Hence we have maps

$$
\mathbb{P}(V \oplus W) \stackrel{b}{\leftarrow} B \stackrel{p}{\rightarrow} \mathbb{P}(V) \times \mathbb{P}(W),
$$

where $b$ is proper and $p$ is flat of relative dimension 1 .

Claim 3.7. Let $\mu: z_{r}(\mathbb{P}(V)) \times z_{s}(\mathbb{P}(W)) \rightarrow z_{r+s}(\mathbb{P}(V) \times \mathbb{P}(W))$ denote the bilinear extension of the map that sends irreducible subvarieties $Z \subset \mathbb{P}(V)$ and $Z^{\prime} \subset \mathbb{P}(W)$ to $Z \times Z^{\prime}$. Then $\mu$ is a continuous map. 
Proof. (of Claim) We use the "flat families" description of the cycle spaces, cf. Appendix 2. If $\Gamma \in \mathcal{Z}_{r}(\mathbb{P}(V) / S)^{\mathrm{fl}}$ and $\Gamma^{\prime} \in \mathcal{Z}_{s}\left(\mathbb{P}(W) / S^{\prime}\right)^{\mathrm{fl}}$ are flat cycles over smooth varieties $S$ and $S^{\prime}$, then the product cycle $\Gamma \times \Gamma^{\prime}$ is flat over $S \times S^{\prime}$. Since $\mu \circ\left(\pi_{\Gamma} \times \pi_{\Gamma^{\prime}}\right)=\pi_{\Gamma \times \Gamma^{\prime}}$, where the $\pi_{-}$'s are defined Example 2.4, one concludes that $\mu$ is continuous.

It is easy to show that

$$
\sigma \# \sigma^{\prime}=b_{*} \circ p^{*} \circ \mu\left(\sigma, \sigma^{\prime}\right) .
$$

This expresses the join pairing as the composition of a proper push-forward, a flat pull-back and a continuous pairing; as shown in the Claim and Property 3.

Remark 3.8. The proof of continuity of the join pairing, used in [FM94] for example, uses Chow varieties and the notion of algebraic continuous maps. Subsequently, Barlet and (independently) J. Plümer showed that the join of cycles induces an algebraic map on the level of Chow varieties. The description of the join pairing given here appeared in [LF93b].

3.2.2. Lawson's suspension theorem. Using the join of algebraic cycles one can define Lawson's complex suspension homomorphism as follows.

Definition 3.9. Given a projective variety $X \subset \mathbb{P}(V)$, let $\Varangle X \subset \mathbb{P}(V \oplus \mathbb{C})$ denote the projective cone $X \# x_{\infty}$ over $X$, where $x_{\infty}:=\mathbb{P}(0 \oplus \mathbb{C}) \in \mathbb{P}(V \oplus \mathbb{C})$. Extending this construction linearly to cycles supported on $X$, one obtains a continuous homomorphism:

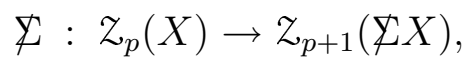

called the (complex) algebraic join homomorphism.

Remark 3.10. Observe that ${ }^{2} \& X$ and $L:=\notin X-\left\{x_{\infty}\right\}$ are, respectively, the Thom space and the total space of the hyperplane bundle associated to $\mathcal{O}_{X}(1)$ over $X$. Furthermore, since a $(p+1)$ cycle cannot be supported on the vertex $x_{\infty}$ of the cone, one has identifications

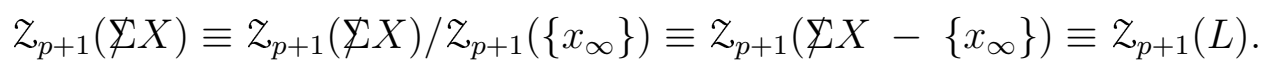

Using the "Chow varieties description" of the topology on cycles (cf. Definition 2.13) one sees that the identities above are topological group isomorphisms. Under this identification, the "suspension homomorphism" is simply the flat pull-back $\rho^{*}: z_{p}(X) \rightarrow z_{p+1}(L)$ under the bundle projection $\rho: L \rightarrow X$. We must point out, however, that Lawson's work [Law89] preceded the introduction of the topological structure of cycles on quasiprojective varieties [LF92].

Theorem 3.11 ([Law89]). For any projective variety $X$, the algebraic suspension homomorphism $\&: z_{p}(X) \rightarrow z_{p+1}(\Varangle X)$ is a homotopy equivalence. Equivalently, pull-back induces a homotopy equivalence $z_{p}(X) \rightarrow z_{p+1}(L)$.

\footnotetext{
${ }^{2}$ In this assertion we are actually identifying $\Varangle X$ and $\notin X(\mathbb{C})$ with the analytic topology.
} 
Corollary 3.12. For any projective variety $X \subset \mathbb{P}(W)$, and complex vector space $V$ of dimension $v$, the join homomorphism $\mathbb{P}(V) \#(-): z_{p}(X) \rightarrow z_{p+v}(\mathbb{P}(V) \# X)$ is a homotopy equivalence.

Using Property 4 one can prove the following homotopy property for Lawson homology.

Corollary 3.13 (Homotopy Property [FG93]). Let $\rho: E \rightarrow X$ be an algebraic vector bundle of rank e over an algebraic variety $X$. Then the flat pull-back $\rho^{*}: z_{p}(X) \rightarrow z_{p+e}(E)$ is a homotopy equivalence.

Proof. (Sketch) The first step is to show that the corollary holds for the trivial line bundle over any affine variety $Y$. This follows from Theorem 3.11 along with Noetherian induction and localization exact sequences.

Once this is shown, one can use induction on rank of the bundle and prove the corollary for any trivial vector bundle over an affine variety $Y$. Using localization sequences and Noetherian induction once again, one then proves the corollary for an arbitrary variety $X$.

Example 3.14. As an immediate consequence of Lawson's theorem, one can compute the Lawson homology of projective spaces $\mathbb{P}^{N}=\mathbb{P}\left(\mathbb{C}^{N+1}\right)=\mathbb{P}\left(\mathbb{C}^{p} \oplus \mathbb{C}^{N-p+1}\right)=\mathbb{P}\left(\mathbb{C}^{p}\right) \# \mathbb{P}^{N-p}$, for $0 \leq p \leq N$. Corollary 3.12 gives a homotopy equivalence $z_{0}\left(\mathbb{P}^{N-p}\right) \cong z_{p}\left(\mathbb{P}^{N}\right)$. This yields isomorphisms

$$
L_{p} H_{n}\left(\mathbb{P}^{N}\right):=\pi_{n-2 p}\left(z_{p}\left(\mathbb{P}^{N}\right)\right) \cong \pi_{n-2 p}\left(z_{0}\left(\mathbb{P}^{N-p}\right)\right) \cong H_{n-2 p}\left(\mathbb{P}^{N-p} ; \mathbb{Z}\right),
$$

where the latter one comes from Theorem 3.3(b).

\subsubsection{Exercises.}

Exercise 3.1. Prove the assertions in Remark 3.4.

Exercise 3.2. Prove that the algebraic join is given by the composition $\sigma \# \sigma^{\prime}=b_{*} \circ p^{*} \circ \mu\left(\sigma, \sigma^{\prime}\right)$, as described in (5).

Exercise 3.3. Prove Corollary 3.12.

Exercise 3.4. Write the details of the proof of Corollary 3.13.

Exercise 3.5. Given an algebraic variety $X$ and $x_{\infty} \in \mathbb{P}^{1}$, one has two maps $i_{\infty}: Z_{r+1}(X) \rightarrow$ $z_{r+1}\left(X \times \mathbb{P}^{1}\right)$ and $p_{1}^{*}: z_{r}(X) \rightarrow z_{r+1}\left(X \times \mathbb{P}^{1}\right)$, where the first is induced by the inclusion and the latter is a flat pull-back by the proper projection $p_{1}: X \times \mathbb{P}^{1} \rightarrow X$. Prove that the map

$$
\begin{aligned}
\psi: z_{r}(X) \times z_{r+1}(X) & \rightarrow z_{r+1}\left(X \times \mathbb{P}^{1}\right) \\
(\sigma, \tau) & \mapsto i_{\infty}(\sigma)+p_{1}^{*} \tau
\end{aligned}
$$

is a homotopy equivalence. (This is a baby version of the projective bundle formula proven in [FG93].) 
HINT: Consider the commutative diagram

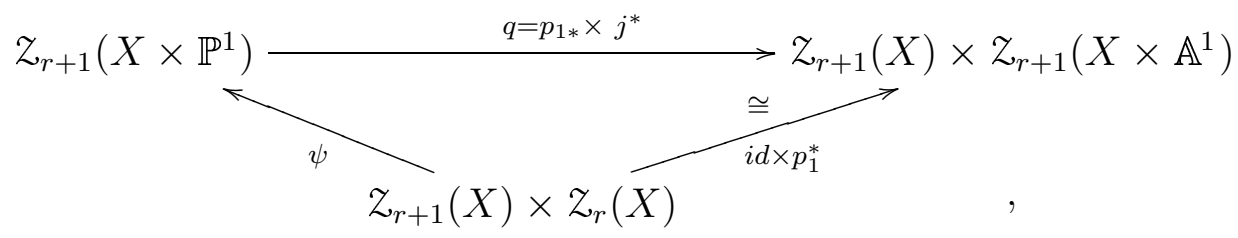

where $j^{*}: Z_{r+1}\left(X \times \mathbb{P}^{1}\right) \rightarrow Z_{r+1}\left(X \times \mathbb{A}^{1}\right)$ is the restriction map induced by the inclusion $j: \mathbb{A}^{1}=\mathbb{P}^{1}-\left\{x_{\infty}\right\} \hookrightarrow \mathbb{P}^{1}$. Then, use the localization sequence for the pair $\left(X \times \mathbb{P}^{1}, X \times\left\{x_{\infty}\right\}\right)$ to show that the map $q$ in the diagram is a homotopy equivalence.

Remark 3.15. Note that this exercise shows the following:

a: $L_{r+1} H_{n}\left(X \times \mathbb{P}^{1}\right) \cong L_{r+1} H_{n}(X) \oplus L_{r} H_{n-2}(X)$;

b: The kernel of the homomorphism $L_{r+1} H_{n}\left(X \times \mathbb{P}^{1}\right) \rightarrow L_{r+1} H_{n}(X)$ is the direct summand of $L_{r+1} H_{n}\left(X \times \mathbb{P}^{1}\right)$ given as the image of the flat pull-back homomorphism

$$
L_{r} H_{n-2}(X) \rightarrow L_{r+1} H_{n-2}\left(X \times \mathbb{P}^{1}\right) .
$$

\section{First APPLICATIONS}

In this section we present a quick overview of some applications that can be immediately derived from the basic properties of algebraic cycles functors and Lawson homology.

4.1. The $s$-map and cycle maps. Using the join pairing and Lawson's suspension theorem, E. Friedlander and B. Mazur constructed a set of operations on the Lawson homology of a projective variety $X$ that yields interesting filtrations on both the ordinary homology of $X$ and on the groups $z_{p}(X)$. Here we present only the operation called the s-map.

Consider the composition $\mathbb{P}^{1} \times z_{p}(X) \stackrel{u \times i d}{\longrightarrow} z_{0}\left(\mathbb{P}^{1}\right)_{o} \times z_{p}(X) \stackrel{\#}{\rightarrow} z_{p+1}\left(\mathbb{P}^{1} \# X\right)_{o}$, where $u: \mathbb{P}^{1} \rightarrow Z_{0}\left(\mathbb{P}^{1}\right)_{o}$ is the inclusion sending $x \in \mathbb{P}^{1}$ to $x-x_{\infty}$, where $x_{\infty}=\mathbb{P}(0 \oplus \mathbb{C})$ is a fixed base-point in $\mathbb{P}^{1}=\mathbb{P}(\mathbb{C} \oplus \mathbb{C})$. It is clear that this map factors through $\mathbb{P}^{1} \wedge z_{p}(X)$ and fits into the following diagram.

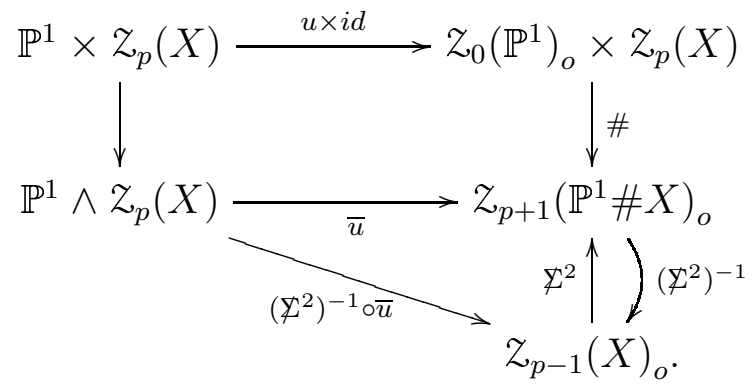


Let

$$
\sigma: z_{p}(X) \rightarrow \Omega^{2} z_{p-1}(X)_{o}
$$

be the unique homotopy class of maps given as the adjoint of the composition $\left(Z^{2}\right)^{-1} \circ \bar{u}$, where $\left(\mathbb{Z}^{2}\right)^{-1}$ is a homotopy inverse for $\mathbb{Z}^{2}$. Therefore, $\sigma$ induces homomorphisms $\sigma_{*}: \pi_{r}\left(Z_{p}(X)\right) \rightarrow$ $\pi_{r+2}\left(z_{p-1}(X)\right)$, and for $r=n-2 p$ this yields the following.

Definition 4.1 ([FM94]). Let $X$ be a projective variety.

a: The $s$-map $s: L_{p} H_{n}(X) \rightarrow L_{p-1} H_{n}(X)$ is the map induced by $\sigma: Z_{p}(X) \rightarrow$ $\Omega^{2} z_{p-1}(X)_{o}$ on Lawson homology.

b: The $p$-th successive composition induces the cycle map for projective varieties:

$$
s^{p}: L_{p} H_{n}(X) \rightarrow L_{0} H_{n}(X) \equiv H_{n}(X ; \mathbb{Z}) .
$$

See Theorem 3.3(b).

Remark 4.2. One must note that both the $s$-map and the cycle map depend a priori on the projective embedding. We shall see shortly that this is not the case.

\section{Digression on the cycle map:}

Using geometric measure theory (Lawson's initial approach to the subject), one can give an alternative description of the cycle map, which proves its functorial nature and consequent independence from embeddings. Let $\mathcal{J}_{k}(X)$ denote the group of integral $k$-currents [Fed69] on the analytic space $X$, with the flat-norm topology. Generalizing the classical Dold-Thom theorem, F. Almgren [Alm62] exhibited a natural isomorphism (holds for any compact ANR)

$$
\Phi: \pi_{r}\left(\mathcal{J}_{k}(X)\right) \cong H_{r+k}(X ; \mathbb{Z}) .
$$

For a projective variety, Lawson showed that the group $Z_{p}(X)$ (Chow topology) sits inside $\mathcal{J}_{2 p}(X)$ as a closed subgroup. The following theorem provided the first proof that the cycle map is natural. In particular that it is independent from the embedding.

Theorem 4.3 ([LF93b]). The composition

$$
L_{p} H_{n}(X):=\pi_{n-2 p}\left(Z_{p}(X)\right) \rightarrow \pi_{n-2 p}\left(\mathcal{J}_{2 p}(X)\right) \stackrel{\Phi}{\rightarrow} H_{n}(X ; \mathbb{Z})
$$

coincides with Friedlander-Mazur cycle maps $s^{p}$.

This result also shows that the cycle map is compatible with localization exact sequences.

The $s$-map has a somewhat simpler description, once one uses the various properties described in previous sections. The following result, for quasiprojective varieties, was shown in [FG93] and [Fri95]. The proof presented here avoids the intersection theory machinery used in the original papers. 
Proposition 4.4. Given a projective algebraic variety $X$ and $p \geq 1$, the homotopy class of the map $\sigma: Z_{p}(X) \rightarrow \Omega^{2} z_{p-1}(X)_{o}$ is given by the adjoint of the composition

$$
\mathbb{P}^{1} \wedge z_{p}(X) \stackrel{\bar{u}}{\rightarrow} z_{0}\left(\mathbb{P}^{1}\right)_{o} \times z_{p}(X) \stackrel{\mu}{\rightarrow} z_{p}\left(X \times \mathbb{P}^{1}\right)_{o} \stackrel{j^{*}}{\rightarrow} z_{p}\left(X \times \mathbb{A}^{1}\right)_{o} \stackrel{\rho^{*-1}}{\longrightarrow} z_{p-1}(X)_{o},
$$

where $\bar{u}$ is defined in Diagram (7), $\mu$ is the product of cycles, $j: X \times \mathbb{A}^{1} \hookrightarrow \mathbb{P}^{1}$ is the inclusion and $\rho: X \times \mathbb{A}^{1} \rightarrow X$ is the projection.

Proof. We denote by $E$ the total space of the bundle associated to $p_{1}^{*} \mathcal{O}_{\mathbb{P}^{1}}(-1) \oplus p_{2}^{*} \mathcal{O}_{X}(-1)$, and refer the reader to Exercise 3.5 and (7) for additional notation. The following diagram summarizes the maps involved in the proof, where $p r_{1}, p_{1}, i_{1}$ and $\iota_{1}$ denote the evident projections and inclusions, respectively.

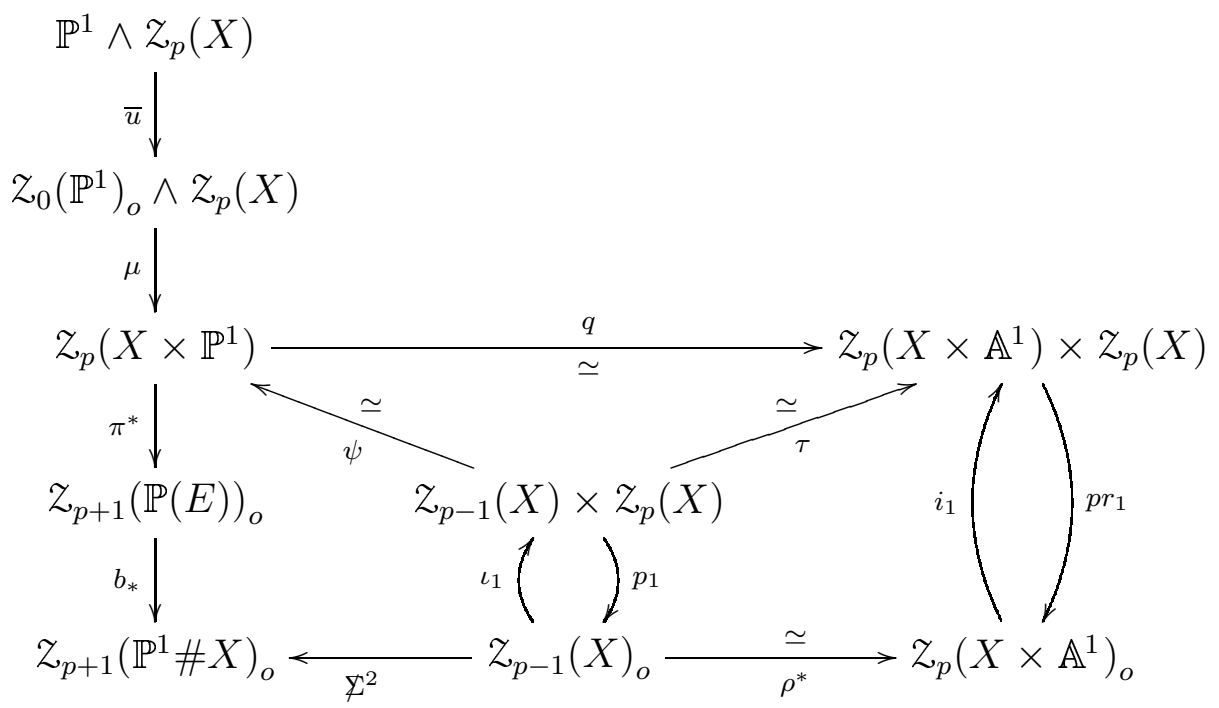

We want to show that the composition $\left(\mathbb{K}^{2}\right)^{-1} \circ b_{*} \circ \pi^{*} \circ \mu \circ \bar{u}$ is homotopic to $\left(\rho^{*}\right)^{-1} \circ j^{*} \circ \mu \circ \bar{u}$. Since $j^{*}=p r_{1} \circ q$, we will show:

$$
b_{*} \circ \pi^{*} \circ \mu \circ \bar{u} \simeq \mathbb{Z}^{2} \circ\left(\rho^{*}\right)^{-1} \circ p r_{1} \circ q \circ \mu \circ \bar{u} .
$$

First we write

$$
\Varangle^{2}=b_{*} \circ \pi^{*} \circ \psi \circ \iota_{1}
$$

and hence the second term of (10) can be written as

$$
b_{*} \circ \pi^{*} \circ \psi \circ\left(\iota_{1} \circ\left(\rho^{*}\right)^{-1}\right) \circ p r_{1} \circ q \circ \mu \circ \bar{u}=b_{*} \circ \pi^{*} \circ \psi \circ\left(\tau^{-1} \circ i_{1}\right) \circ p r_{1} \circ q \circ \mu \circ \bar{u}
$$

where the equality comes from the fact that $i_{1} \circ \rho^{*}=\tau \circ \iota_{1}$; see diagram above.

The main observation now is the fact that the image of $\mu: z_{0}(X)_{o} \times z_{p}(X) \rightarrow z_{p}\left(X \times \mathbb{P}^{1}\right)$ lies in the kernel of the map $z_{p}\left(X \times \mathbb{P}^{1}\right) \rightarrow z_{p}(X)$. Hence the image of the composition $q \circ \mu \circ \bar{u}$ 
lies in the kernel of $p r_{2}: z_{p}\left(X \times \mathbb{A}^{1}\right) \times z_{p}(X) \rightarrow z_{p}(X)$. It follows that $q \circ \mu \circ \bar{u}=$ $i_{1} \circ p r_{1} \circ q \circ \mu \circ \bar{u}$. Applying this identity to (12), along with the fact that $\psi \circ \tau^{-1} \circ q \simeq i d$, one obtains (10).

Corollary 4.5. The s-map is a functorial construction, and hence, it is independent of the embedding.

Remark 4.6. Friedlander and Gabber [FG93] used their intersection theory for Lawson homology to prove the above proposition in the context derived categories.

Definition 4.7. For an arbitrary algebraic variety $X$, let $\sigma: z_{p}(X) \rightarrow \Omega^{2} z_{p-1}(X)_{o}$ be defined as in Proposition 4.4. In the level of homotopy groups, this defines the $s$-map $s: L_{p} H_{n}(X) \rightarrow$ $L_{p-1} H_{n}(X)$ and the cycle map $s^{p}: L_{p} H_{n}(X) \rightarrow L_{0} H_{n}(X) \cong H_{n}^{B M}(X ; \mathbb{Z})$, for an arbitrary variety $X$.

\subsubsection{Exercises.}

Exercise 4.1. Prove that the image of the map $z_{0}\left(\mathbb{P}^{1}\right)_{o} \times z_{p}(X) \stackrel{\mu}{\rightarrow} z_{p}\left(X \times \mathbb{P}^{1}\right)$ lies in the kernel of the map $z_{p}\left(X \times \mathbb{P}^{1}\right) \rightarrow z_{p}(X)$.

Exercise 4.2. Prove that the $s$-map is compatible with localization sequences (for arbitrary varieties).

4.2. Friedlander-Mazur filtrations on cycles. Using the $s$-map, Friedlander and Mazur constructed quite interesting filtrations on cycle spaces of projective varieties in [FM94]. Subsequently, these filtrations were extended to quasiprojective varieties in [Fri95]. We describe these filtrations below, where we also show that they can be defined for arbitrary complex varieties.

Recall that

$$
L_{p} H_{2 p}(X)=\pi_{0}\left(z_{p}(X)\right) \cong z_{p}(X) / \mathcal{z}_{p}(X)_{o}=\mathcal{A}_{p}(X),
$$

cf. Theorem 3.3(b). In particular one has a composition

$$
z_{p}(X) \stackrel{\pi}{\rightarrow} z_{p}(X) / z_{p}(X)_{o} \cong L_{p} H_{2 p}(X) \stackrel{s^{j}}{\rightarrow} L_{p-j} H_{2 p}(X),
$$

where $\pi$ denote the projection and $s^{j}$ is the $j$-th iteration of the cycle map.

Definition 4.8. Define the $j$-th stage of the topological filtration on $z_{p}(X)$ as $S_{j} z_{p}(X):=$ ker $s^{j} \circ \pi$. Note that this forms a filtration

$$
z_{p}(X)_{\mathrm{alg}} \equiv S_{0} z_{p}(X) \subseteq S_{1} z_{p}(X) \subseteq \cdots \subseteq S_{p} z_{p}(X) \equiv z_{p}(X)_{\mathrm{hom}},
$$

interpolating between the group of cycles algebraically equivalent to zero $z_{p}(X)_{\text {alg }}$ and $z_{p}(X)_{\text {hom }}$, the group of cycles homologically equivalent to zero, i.e., the kernel of the cycle map.

Remark 4.9. Taking quotients by $z_{p}(X)_{\text {alg }}$ one obtains a filtration of the Griffiths group

$$
z_{p}(X)_{\mathrm{hom}} / z_{p}(X)_{\mathrm{alg}}
$$


This filtration is given a different formulation in [Fri95], using the notion of Chow correspondences, a generalization of the usual notion of correspondences. Roughly speaking, a Chow correspondence $f: Y \rightarrow \mathcal{C}_{p, \alpha}(X)$ is a morphism between the weak normalization of a variety $Y$ to the weak normalization of a Chow variety $\mathcal{C}_{p, \alpha}(X)$ of $p$-cycles on a projective variety $X$. (This is also called an algebraic continuous map.) One can show that such a correspondence induces a continuous graphing map $\Gamma_{f}: z_{r}(X) \rightarrow z_{p+r}(X)$. See [Fri95] for details.

Theorem 4.10 ([Fri95]). For any projective algebraic variety $X, S_{j} z_{p}(X) \subseteq z_{p}(X)$ is the subgroup generated by cycles $Z$ of the following form: there exists a projective variety $Y$ of dimension $2 j+1$, a Chow correspondence $f: Y \rightarrow \mathcal{C}_{p-j}(X)$, and a $j$-cycle $W$ on $Y$ homologically equivalent to 0 in $Y$ and such that $Z$ is rationally equivalent to $\Gamma_{f}(W)$.

Among the many interesting properties of this filtration is the fact that, when $X$ is smooth, it interpolates between Nori's filtration [Nor93] and Bloch-Ogus' filtration [BO84]. These filtrations are roughly defined as follows.

The $j$-th level $A_{j} C H_{p}(X)$ of Nori's filtration is defined as the subgroup generated by those algebraic cycles that are rationally equivalent to cycles of the form $p r_{X *}\left(p r_{Y}^{*} X \cdot Z\right)$, where $Y$ is a smooth projective variety, $W \in z_{j}(Y)_{\text {hom }}$ and $Z \in z_{p+\operatorname{dim} Y-j}(Y \times X)$.

Similarly, the $j$-th level $B_{j} C H_{p}(X)$ of Bloch-Ogus filtration is the subgroup generated by the $p$-cycles $c$ on $X$ such that $c$ is supported on a subvariety $V$ of $X$ of dimension $p+j+1$ and $c$ is homologically equivalent to zero in $V$.

Theorem 4.11 ([Fri95]). If $X$ is a smooth projective variety, then one has inclusions

$$
A_{j} C H_{p}(X) \subseteq S_{j} z_{p}(X) \subseteq B_{j} C H_{p}(X)
$$

where $A_{j} C H_{p}(X)$ is Nori's filtration on $z_{p}(X)$ and $B_{j} C H_{p}(X)$ is Bloch-Ogus's.

Question 4.12. Let $(V, \sigma)$ be a pair consisting of a complex variety $V$ and a $p$-cycle $\sigma \in$ $z_{p}(V)_{\text {hom }}$, homologically equivalent to zero. Under which conditions can one find a smooth variety $\widehat{V}$ along with a $p$-cycle $\widehat{\sigma}$ on $\widehat{V}$ and a proper map $f: \widehat{V} \rightarrow V$ such that $f_{*}(\widehat{\sigma})=\sigma$ and $\widehat{\sigma}$ is also homologically equivalent to zero in $\widehat{V}$ ? If this question has a positive answer under general circumstances, then Nori's and Friedlander's filtrations coincide.

\section{5. (COlimits of) MiXed Hodge Structures on LAWSON HOMOlogy}

In [FM94], Friedlander and Mazur showed that one can naturally endow the Lawson homology of projective varieties with the structure of a "colimit" of Mixed Hodge structures". Here we provide an alternative construction that can also be applied to arbitrary varieties, and we show that this construction coincides with the one in [FM94] in the projective case. 


\subsection{A Brief Review of Mixed Hodge Structures.}

A (pure) Hodge structure of weight $m$ on a finite dimensional real vector space $V$ is a decreasing filtration

$$
\cdots \subset F^{p+1} V_{\mathbb{C}} \subset F^{p} V_{\mathbb{C}} \subset \cdots
$$

of the complexified vector space $V_{\mathbb{C}}=V \otimes_{\mathbb{R}} \mathbb{C}$ satisfying the Hodge decomposition

$$
V_{\mathbb{C}}=\bigoplus_{p+q=m} V^{p, q}
$$

where $V^{p+q}=F^{p} V_{\mathbb{C}} \cap \overline{F^{q} V_{\mathbb{C}}}$ and $\overline{F^{*} V_{\mathbb{C}}}$ is the conjugate filtration.

If $A$ is a subring of $\mathbb{R}$, an $A$-Hodge structure of weight $m$ is an $A$-module $V_{A}$ of finite type together with a Hodge structure of weight $m$ on $V_{\mathbb{R}}=V_{A} \otimes_{A} \mathbb{R}$.

Exercise 5.1. $\quad$ a) Show that if $V$ and $W$ carry Hodge structures of weights $m, n$ respectively, then $V \otimes W$ carries a Hodge structure of weight $m+n$.

b) (The Tate Hodge structure) Prove that $\mathbb{Z}(1):=(2 \pi i) \mathbb{Z} \subset \mathbb{C}$ has a unique $\mathbb{Z}$-Hodge structure of weight -2 . Denote by $\mathbb{Z}(n)$ the $\mathbb{Z}$-Hodge structure $\mathbb{Z}(n):=\mathbb{Z}(1) \otimes \cdots \otimes \mathbb{Z}(1)$ (of weight $-2 n$ ).

Remark 5.1. In the ground-breaking work [Hod41] on the theory of harmonic integrals, Hodge showed that the cohomology group $H^{m}(X ; \mathbb{R})$ of a compact Kähler manifold $X$ carries a Hodge structure of weight $m$.

Definition 5.2. Given a subring $A \subset \mathbb{R}$, an $A$-mixed Hodge structure (MHS) consists of:

(1) An $A$-module of finite type $V_{A}$;

(2) An increasing filtration

$$
\cdots \subset W_{n} \subset W_{n+1} \subset \cdots
$$

of the $A \otimes \mathbb{Q}$ module $V_{A} \otimes \mathbb{Q}$;

(3) A decreasing filtration

$$
\cdots \subset F^{p+1} \subset F^{p} \subset \cdots
$$

of $V_{\mathbb{C}}=V_{A} \otimes \mathbb{C}$.

These filtrations must satisfy the following property. If one defines $G r_{j}^{W}\left(V_{\mathbb{C}}\right)=\left(W_{j} \otimes \mathbb{C}\right) /\left(W_{j-1} \otimes\right.$ $\mathbb{C})=\left(W_{j} / W_{j-1}\right) \otimes \mathbb{C}$, then the filtrations $F$ and $\bar{F}$ on $V_{\mathbb{C}}$ induce an $A$-Hodge structure of weight $j$ on $G r_{j}^{W}\left(V_{A}\right):=W_{j} / W_{j-1}$.

A morphism $f: V_{A} \rightarrow V_{A^{\prime}}$ of $A$-MHS is an $A$-module homomorphism which is compatible with both filtrations $W$ and $F$. With this notion, one has a category of $A$-mixed Hodge structures.

Theorem 5.3. [Del71, Th. 2.3.5] 
(1) The category of A-MHS is abelian, with kernels and cokernels of morphisms endowed with the induced filtrations;

(2) The functor $V \mapsto G r_{j}^{W}(V)$ is an exact functor from the category of A-MHS to the category of A-HS of weight $j$;

(3) The functor $V \mapsto G r_{F}^{p}(V)$ is exact.

In [Del71] Deligne generalized Hodge's result to include arbitrary smooth complex algebraic varieties.

Theorem 5.4. [Del71, Th. 3.2.5] There are filtrations $W[p]$ and $F$ which define on $H^{p}(X ; \mathbb{Z}) a$ mixed Hodge structure. This MHS is functorial with respect to algebraic maps.

Subsequently, in [De174], Deligne went even further and proved the existence of MHS on the cohomology of arbitrary complex varieties. In order to construct this MHS, one needs to resort to simplicial varieties. In fact, for any simplicial scheme $X$ • over $\mathbb{C}$, one can construct [Del74, $\S 8.3]$ a structure of MHS on the cohomology groups $H^{*}\left(X_{\bullet} ; \mathbb{Z}\right)$, and this structure is functorial on $X_{\bullet}$.

Recall that a simplicial variety over $\mathbb{C}$ consists of a collection $\left\{X_{n} ; d_{i}, s_{i}\right\}$ of complex algebraic varieties $X_{n}, n=0,1,2, \ldots$, along with morphisms $\delta_{i}: X_{n} \rightarrow X_{n-1}, i=0, \ldots, n$ (the "face maps") and morphisms $s_{i}: X_{n} \rightarrow X_{n+1}, i=0, \ldots, n$ satisfying certain compatibility relations; see [May82] for basics on simplicial objects.

Example 5.5. Let $G$ be a complex algebraic group acting algebraically on a variety $X$. Then the bar construction (see $\S 2.3) \mathcal{B} \bullet(X, G, *)$ with $n$-th variety $\mathcal{B}_{n}(X, G, *)=X \times G \times \cdots \times G$ (and whose face and degeneracy maps are defined in $\S 2.3$ ) is an example of a simplicial variety. The particular cases $E_{\bullet} G:=\mathcal{B}_{\bullet}(G, G, *)$ and $B_{\bullet} G:=\mathcal{B}(*, G, *)$ give simplicial varieties so that the natural map $E_{\bullet} G \rightarrow B_{\bullet} G$ is (after geometric realization) the universal principal $G$-bundle over the classifying space $B G$ of $G$. It is easy to see that $\mathcal{B}(X ; G ; *) \cong X \times_{G} E G$ is the usual Borel construction on $X$.

\subsection{Colimits of M.H.S. and Lawson Homology.}

Definition 5.6. Let $A$ be a subring of $\mathbb{R}$. An $A$-module $M_{A}$ is a colimit of $A$-Mixed Hodge structures if there is a directed system $\left\{M_{A, \lambda}\right\}_{\lambda \in \Lambda}$ of $A$-Mixed Hodge structures (i.e. all maps $\varphi_{\mu}^{\lambda}: M_{A, \lambda} \rightarrow M_{A, \mu}$ are maps of $A$-MHS) such that

$$
M_{A}=\underset{\lambda}{\operatorname{colim}} M_{A, \lambda}
$$

The following result is proven in [FM94] in the case of projective varieties. The general case presented here is a novel result. Also, we use a rather different approach, avoiding the use of the (non-canonical) notion of base systems as in [FM94]. 
Theorem 5.7. Let $X$ be a complex algebraic variety and let $z_{p}(X)$ denote the group of algebraic p-cycles on $X$, with the topology defined in Section 2.

a. The homology groups $H_{m}\left(Z_{p}(X) ; \mathbb{Z}\right)$ admit the structure of a colimit of $\mathbb{Z}-M H S$.

b. This structure is covariantly functorial for proper morphisms and contravariant for flat morphisms.

c. The Hurewicz homomorphism

$$
h: L_{p} H_{n}(X)=\pi_{n-2 p}\left(Z_{p}(X)\right) \longrightarrow H_{n-2 p}\left(Z_{p}(X) ; \mathbb{Z}\right)
$$

is injective, hence it gives a functorial structure of colimit of $\mathbb{Z}-M H S$ on Lawson homology.

d. If $X$ is projective, then this structure coincides with the one in [FM94].

Before sketching the proof, let us establish some conventions.

NotATION: If $Y$ is a projective variety and $\mathcal{C}_{p, \alpha}(Y)$ is a Chow variety, let $\nu: \mathcal{e}_{p, \alpha}(Y)^{w} \rightarrow$ $\mathrm{e}_{p, \alpha}(Y)$ denote its weak normalization. This is a homeomorphism of underlying topological spaces and hence it induces isomorphism between the MHS's in their cohomology. The advantage of using $\mathcal{C}_{p, \alpha}(Y)^{w}$ instead of $\mathcal{e}_{p, \alpha}(Y)$ lies in the fact that if $f: X \rightarrow Y$ is morphism of projective varieties, then $f_{*}: \mathcal{C}_{p, \alpha}(X)^{w} \rightarrow \mathcal{C}_{p, f_{*}(\alpha)}(Y)^{w}$ is a morphism between Chow varieties. From now on, we denote $\mathcal{C}_{p, \alpha}(X)^{w}$ simply by $\mathcal{C}_{p, \alpha}(X)$. See [FM94] or [Kol96] for details.

Sketch of Proof (of Theorem 5.7).

We first consider the case $X$ quasiprojective. Let $j: X \hookrightarrow \bar{X}$ be a projective compactification and denote $D=\bar{X}-X$.

Consider $M:=\mathfrak{C}_{p}(\bar{X}) \times \mathfrak{C}_{p}(\bar{X})$, where $\mathfrak{C}_{p}(\bar{X})$ is the Chow monoid of effective $p$-cycles in $\bar{X}$, and denote by $N \subset M$ the image of the map

$$
\begin{aligned}
\mathcal{C}_{p}(D) \times \mathcal{C}_{p}(D) \times \mathcal{C}_{p}(\bar{X}) & \longrightarrow \mathcal{C}_{p}(\bar{X}) \times \mathcal{C}_{p}(\bar{X}) \\
(a b ; \lambda) & \longmapsto(a+\lambda, b+\lambda)
\end{aligned}
$$

\section{Exercise 5.1.}

1. Show that $N$ is a submonoid of $M$.

2. Show that $N \cap\left(\mathcal{e}_{p, \alpha}(\bar{X}) \times \mathcal{e}_{p, \beta}(\bar{X})\right)$ is subvariety of $\mathfrak{e}_{p, \alpha}(\bar{X}) \times \mathcal{e}_{p, \beta}(\bar{X})$ for all $\alpha, \beta$.

main 3 . Show that the monoid quotient $M / N$ is homeomorphic to $z_{p}(\bar{X}) / z_{p}(D) \equiv z_{p}(X)$.

Lemma 5.8. The map $\mathcal{B}(M, N, *) \longrightarrow M / N \equiv z_{p}(X)$ is a quasifibration with contractible fibers homeomorphic to $\mathcal{B}(N, N, *)$. In particular, $\phi: \mathcal{B}(M, N, *) \longrightarrow z_{p}(X)$ is a homotopy equivalence.

Proof. This follows directly from [LF93a]. 
Now, define

$$
\mathcal{B}_{k}(M, N, *)_{\leq d}=\left\{\left(a, b ; n_{1}, \ldots, n_{k} ; *\right) \in M \times N^{\times k} \mid \operatorname{deg} a+\operatorname{deg} b+2 \sum_{i} \operatorname{deg} n_{i} \leq d\right\}
$$

It is easy to see that

a. $\mathcal{B}_{\bullet}(M, N, *)_{\leq d} \subset \mathcal{B} \bullet(M, N, *)$ is a simplicial subspace.

b. Each $\mathcal{B} \cdot(M, N . *)_{\leq d}$ is a simplicial variety and the inclusion

$$
\mathcal{B} \bullet(M, N, *)_{\leq d} \hookrightarrow \mathcal{B} \bullet(M, N, *)_{\leq d+1}
$$

is morphism of simplicial varieties.

Since each $\mathcal{B} \cdot(M, N, *)_{\leq d}$ is a simplicial variety whose geometric realization is a finite $\mathrm{CW}$ complex, its integral homology admits the structure of a $\mathbb{Z}$-MHS via the Kronecker duality pairing $H_{*, \mathbb{Q}} \otimes H_{\mathbb{Q}}^{*} \rightarrow \mathbb{Q}$. This gives $H_{*}$ a unique $\mathbb{Z}$-MHS so that the pairing is a map of MHS when $\mathbb{Q}$ is given the Hodge structure of weight 0 .

We now have $\mathcal{B} \cdot(M, N, *)=\underset{d}{\operatorname{colim}} \mathcal{B}(M, N, \cdot)_{\leq d}$ and, since homology commutes with colimits, one obtains

$$
\begin{aligned}
H_{n}\left(\mathcal{Z}_{p}(X) ; \mathbb{Z}\right) & \cong H_{n}(\mathcal{B}(M, N, *) ; \mathbb{Z})= \\
& =\operatorname{colim}_{d} H_{n}\left(\mathcal{B}(M, N, *)_{\leq d} ; \mathbb{Z}\right),
\end{aligned}
$$

where the latter is a colimit of $\mathbb{Z}$-MHS. This proves statement (a) of the theorem.

Lemma 5.9. Let $G$ be an abelian topological monoid whose topology is given by an increasing sequence $\{0\} \subset G_{1} \subset \cdots \subset G_{d} \subset G_{d+1} \subset \cdots$ of compact subsets. Then the Hurewicz map $h: \pi_{k}(G) \rightarrow H_{k}(G ; \mathbb{Z})$ is injective.

Proof. This result is classical. (The filtration condition is not really needed). Let $1 \in G$ denote the unity of $G$, whose operation we denote multiplicatively. Since $G$ is abelian, the map

$$
\begin{aligned}
& G_{d} \times \cdots \times G_{d} \stackrel{\bullet}{\longrightarrow} G \\
& g_{1}, \ldots, g_{n} \longmapsto g_{1} \cdot \ldots \cdot g_{n}
\end{aligned}
$$

descends to a map

$$
S P_{n}\left(G_{d}\right) \longrightarrow G,
$$

where $S P_{n}\left(G_{d}\right)=\left(G_{d} \times \cdots \times G_{d}\right) / S_{n}$ is the $n$-fold symmetric product. This is compatible with the inclusion 


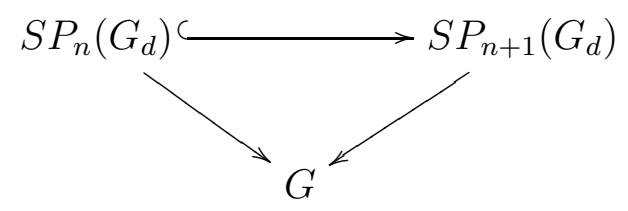

As a result, one gets a map $S P_{\infty}\left(G_{d}\right) \rightarrow G$ having the property that $G_{d} \hookrightarrow S P_{\infty}\left(G_{d}\right) \rightarrow$ $G$ is simply the inclusion $G_{d} \hookrightarrow G$. Now, the Dold-Thom Theorem gives an isomorphism $\pi_{k}\left(S P_{\infty}\left(G_{d}\right)\right) \cong H_{k}\left(G_{d}, \mathbb{Z}\right)$ such that the composition

$$
\pi_{k}\left(G_{d}\right) \longrightarrow \pi_{k}\left(S P_{\infty}\left(G_{d}\right)\right) \cong H_{k}\left(G_{d} ; \mathbb{Z}\right)
$$

is the Hurewicz map. We obtain a map

$$
r: H_{k}(G ; \mathbb{Z})=\operatorname{colim}_{d} H_{k}\left(G_{d} ; \mathbb{Z}\right) \cong \operatorname{colim}_{d} \pi_{k}\left(S P_{\infty}\left(G_{d}\right)\right) \longrightarrow \pi_{k}(G)
$$

so that the composition

$$
\pi_{k}(G)=\underset{d}{\operatorname{colim}} \pi_{k}\left(G_{d}\right) \stackrel{h}{\rightarrow} \underset{d}{\operatorname{colim}} H_{k}\left(G_{d} ; \mathbb{Z}\right) \stackrel{r}{\rightarrow} \pi_{k}(G)
$$

is the identity. It follows that $h$ is injective.

Proof of (c): As a consequence of Lemma 5.9 one has a natural inclusion

$$
h: L_{p} H_{n}(X):=\pi_{n-2 p}\left(z_{p}(X)\right) \hookrightarrow H_{n-2 p}\left(z_{p}(X) ; \mathbb{Z}\right) .
$$

Since the category of colimits of MHS is abelian, we conclude that $L_{p} H_{n}(X)$ inherits a natural structure of colimits of MHS which is clearly functorial.

Proof of (d): If $X$ is projective then $M=\mathfrak{C}_{p}(X) \times \mathcal{C}_{p}(X)$ and $N=\Delta_{\mathfrak{C}_{p}(X)}$ is the diagonal; cf. (14). Choose a base system $\left\{\mathcal{C}_{p, \alpha}(X), z_{\alpha}^{0}\right\}, Z_{\alpha}^{0} \in \mathcal{C}_{p, \alpha}(X)$, where $\alpha$ runs over all connected components of the Chow monoid $\mathrm{C}_{p}(X)$. The map

$$
\begin{aligned}
\iota_{\alpha}: \mathcal{C}_{p, \alpha}(X) & \longrightarrow z_{p}(X)_{0} \\
\sigma & \longmapsto \sigma-z_{\alpha}^{0}
\end{aligned}
$$

fits into a commutative diagram

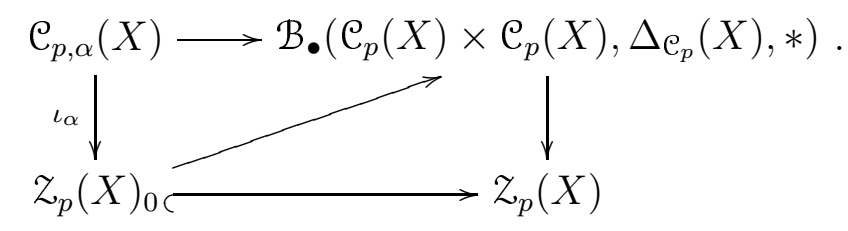


Hence $H_{k}\left(\mathfrak{C}_{p, \alpha}(X) ; \mathbb{Z}\right) \longrightarrow H_{k}\left(Z_{p}(X)_{0} ; \mathbb{Z}\right)$ is a map of (co)limits of MHS. On the other hand, general properties of homotopy theoretic group completions give an isomorphism

$$
\underset{\alpha}{\operatorname{colim}} H_{k}\left(\mathfrak{C}_{p, \alpha}(X), \mathbb{Z}\right) \cong H_{k}\left(\mathcal{Z}_{p}(X)_{0} ; \mathbb{Z}\right)
$$

cf. [FM94]. The previous colimit is used to define the colimit of MHS structure in [FM94].

Exercise 5.2. Using Chow covers, as in Section 2.2, extend the proof above to include the case of arbitrary (not necessarily quasiprojective) varieties.

This concludes the (fairly complete) Sketch of Proof of Theorem 5.7.

The constructions presented here satisfy various other properties that we leave aside in these notes. See [FM94]. Here are some additional results.

Proposition 5.10. Let $X$ be an arbitrary complex variety.

(1) The Pontrjagin ring structure on the homology $H_{*}\left(Z_{p}(X) ; \mathbb{Z}\right)$ becomes a Hopf algebra in the category of colimits of $\mathbb{Z}$-Mixed Hodge structures.

(2) The s-map $s: L_{p} H_{n}(X) \rightarrow L_{p-1} H_{n}(X)$ is in fact a map of colimits of $\mathbb{Z}$-MHS:

$$
S: L_{p} H_{n}(X) \rightarrow L_{p-1} H_{n}(X)(-1):=L_{p} H_{n}(X) \otimes \mathbb{Z}(-1)
$$

Remark 5.11. A slight modification of Milnor-Moore's Theorem allows one to identify the rational homotopy groups $\pi_{*}\left(Z_{p}(X)\right) \otimes \mathbb{Q}$ with the primitives of the Hopf-algebra $H_{*}\left(Z_{p}(X), \mathbb{Q}\right)$, via the Hurewicz homomorphism; cf. [FM94]. This would give $L_{p} H_{n}(X) \otimes \mathbb{Q}$ colimit of $\mathbb{Q}$-mixed Hodge structures.

Proof. 1. Follows from the fact that in the level of bar constructions the various operations that induce the Hopf-algebra structure are given by algebraic maps.

According to Proposition 4.4, the $s$-map is induced by the sequence of maps

$$
\mathbb{P}^{1} \wedge z_{p}(X) \stackrel{\bar{u}}{\rightarrow} z_{0}\left(\mathbb{P}^{1}\right)_{o} \times z_{p}(X) \stackrel{\mu}{\rightarrow} z_{p}\left(X \times \mathbb{P}^{1}\right)_{o} \stackrel{j^{*}}{\rightarrow} z_{p}\left(X \times \mathbb{A}^{1}\right)_{o} \stackrel{\rho^{*-1}}{\longrightarrow} z_{p-1}(X)_{o}
$$

Since $\pi_{1}^{*}$ is a flat pull-back (and homotopy equivalence) it induces an isomorphism of colimits of $\mathbb{Z}$-MHS in the level of homotopy groups. The maps $u$ and $j$ are also derived from algebraic maps and can be easily seen to induce maps of co-MHS. The composition above then gives a pairing 
of co-MHS:

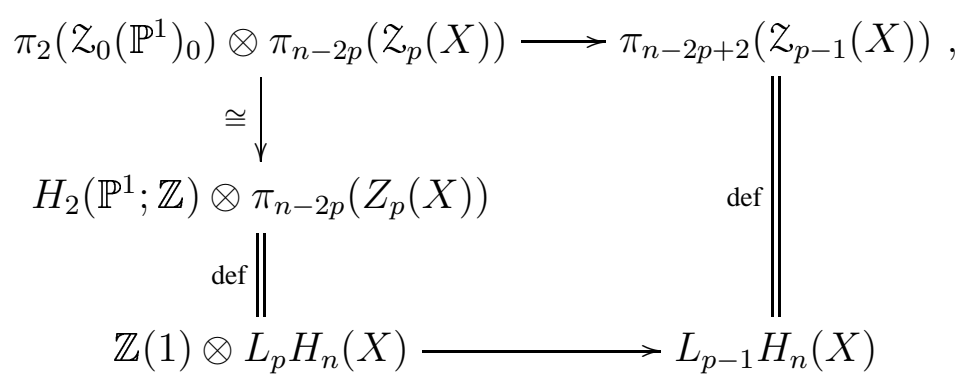

whose adjoint $L_{p} H_{n}(X) \longrightarrow L_{p-1} H_{n}(X) \otimes \mathbb{Z}(-1)$ is precisely the $s$-map.

\subsection{A note on the Abel-Jacobi map.}

Let $X$ be a smooth projective variety over $\mathbb{C}$. Recall that the intermediate Jacobian $g^{k}(X)$ is the complex torus defined by

$$
g^{k}(X):=\frac{H^{2 k-1}(X ; \mathbb{C})}{F^{k} H^{2 k-1}(X ; \mathbb{C})+H^{2 k-1}(X ; \mathbb{Z})} \cong \frac{F^{n-k+1} H^{2 n-2 k+1}(X, \mathbb{C})^{*}}{H^{2 k-1}(X ; \mathbb{Z})^{*}}
$$

The Abel-Jacobi map

$$
\nu: Z^{k}(X)_{\text {hom }} \longrightarrow g^{k}(X)
$$

sends $\sigma \in Z_{\mathrm{hom}}^{k}(X)$ to

$$
\nu(\sigma)=\left[\int_{T} \sigma\right]
$$

where $\sigma=\partial T$ and $T$ is an integral current of dimension $2 n-2 k+1$. Here $\int_{T}$ is the functional on $F^{n-k+1} H^{2 n-2 k+1}(X, \mathbb{C})$ defined by $\int_{T}[\varphi]=T(\varphi)$ and $[-]$ denotes equivalence classes in the appropriate quotient groups. It is easy to see that $\nu$ is well-defined.

Proposition 5.12. Let $X$ be smooth and projective.

(1) $Z^{k}(X)_{\text {hom }}$ is a closed subgroup of $Z^{k}(X)$;

(2) $Z^{k}(X)_{\text {hom }}$ is a union of connected components of $Z^{k}(X)$ and

$$
\pi_{0}\left(z^{k}(X)_{\text {hom }}\right) \cong \frac{z^{k}(X)_{\text {hom }}}{z^{k}(X)_{\text {alg }}} \equiv \underset{\text { group }}{\text { Griffiths }}
$$

(3) The Abel-Jacobi map $\nu: Z^{k}(X)_{\mathrm{hom}} \longrightarrow \mathrm{J}^{k}(X)$ is continuous.

Proof. Assertions (1) and (2) are quite easy to show and we leave them as an exercise. Assertion (3) follows from the continuity (holomorphicity) of normal functions $[\mathrm{K}]$ and the description of the topology on $z^{k}(X)$ in terms of flat families; cf. Section 2. 
5.3.1. Speculations and Remarks. Consider the restriction of the Abel-Jacobi map to the connected component of the identity $z_{1}^{k}(X)_{\mathrm{alg}}=Z^{k}(X)_{0}$ of $Z^{k}(X)$. The image of this restriction is an abelian variety and the kernel is a closed subgroup

$$
F^{1} z^{k}(X)_{0} \subset F^{0} z^{k}(X)_{0} \equiv z^{k}(X)_{\text {alg }}
$$

In particular, $F^{0} / F^{1}$ is a smooth group. On the other hand, the Abel-Jacobi map $\nu$ vanishes on the subgroup $z^{k}(X)_{\text {rat }} \subset Z^{k}(X)_{\text {alg }}$ consisting of the cycles rationally equivalent to zero. Since $\nu$ is continuous, it vanishes on the closure

$$
\overline{z^{k}(X)_{\text {rat }}} \subset z^{k}(X)_{\text {alg }}
$$

Considering generalized intermediate Jacobians $J_{\Phi_{p}}^{k}(X)$ with supports (not exactly same family of supports as in King's work $[\mathrm{K}])$, it is conceivable that one can find a filtration $Z^{k}(X)_{\text {alg }}$ as

$$
z^{k}(X)_{\text {rat }} \subset \bar{z}^{k}(X)_{\text {rat }} \stackrel{?}{\equiv} F^{k+1} \subset \cdots \subset F^{1} \subset F^{0}=Z_{\text {alg }}^{k}
$$

Ideally this should satisfy:

(1) $F^{j} / F^{j+1}$ are (possibly infinite dimensional) smooth groups;

(2) The filtration is functorial;

In particular, the most complicated portion of the topological behavior (i.e. homotopy invariants) of the group of algebraic cycles would lie in the closure of rational equivalence. This would expand Rojtman's work in [Roj71] and [Roj72].

\section{INTERSECTION THEORY}

In this section we present the intersection pairing in Lawson homology, as introduced for quasiprojective varieties by Friedlander and Gabber in [FG93]. Our presentation works for arbitrary varieties, not necessarily quasiprojective. The main ideas are quite universal, and can be applied in many different contexts, relying heavily on the process of deformation to the normal cone. This is a technique introduced by Fulton and MacPherson, and used by Fulton in his beautiful presentation of intersection theory [Ful84].

The main ingredients are the following:

(1) Given algebraic varieties $X, Y$, the product of cycles

$$
z_{p}(X) \times z_{q}(Y) \rightarrow z_{p+q}(X \times Y)
$$

defines a continuous bilinear pairing; cf. Claim 3.7.

(2) Given a closed inclusion $Y \hookrightarrow X$, the sequence

$$
z_{p}(Y) \hookrightarrow z_{p}(X) \rightarrow z_{p}(X-Y)
$$


is a locally trivial fibration. (This is Property 4 in Section 2.)

(3) Given an algebraic vector bundle $E \stackrel{p}{\longrightarrow} X$, the flat pull-back $p^{*}: z_{p}(X) \rightarrow z_{p+e}(E)$ is a homotopy equivalence; cf. Corollary 3.13.

Remark 6.1.

(a) In Fulton's intersection theory, for the Chow groups $C H_{p}(X):=z_{p}(X) / z_{p}(X)$ rat $)$, these conditions have the following counterparts:

(1) Multiplication of cycles preserves rational equivalence;

(2) Given a closed subvariety $Y \subset X$ one has an exact sequence

$$
C H_{p}(Y) \rightarrow C H_{p}(X) \rightarrow C H_{p}(X-Y) \rightarrow 0
$$

(3) Flat-pull back $p^{*}: C H_{p}(X) \rightarrow C H_{p+e}(E)$ is an isomorphism for any vector bundle $E \rightarrow X$.

(b) More generally, for the Higher Chow groups [Blo86] [BL], the following conditions hold. Here $\mathfrak{Z}^{p}(X, *)$ denotes Bloch's complexes.

(1) Multiplication of cycles induces a pairing

$$
\mathfrak{Z}^{p}(X, *) \otimes \mathfrak{Z}^{q}(Y, *) \rightarrow \mathfrak{Z}^{p+q}(X \times Y, *)
$$

in the derived category;

(2) A closed inclusion $Y \hookrightarrow X$ gives a distinguished triangle

$$
\mathfrak{Z}^{p}(Y, *) \rightarrow \mathfrak{Z}^{p+e}(X, *) \rightarrow \mathfrak{Z}^{p+e}(X-Y, *)
$$

in the derived category $(e=\operatorname{dim} X-\operatorname{dim} Y)$. This yields the localization sequences.

(3) Homotopy property:

$$
\mathfrak{Z}^{p}(X, *) \rightarrow \mathfrak{Z}^{p}(E, *)
$$

is a quasi-isomorphism.

\subsection{Deformation to the Normal Cone and Gysin Homomorphism.}

Let $Y \underset{j}{\hookrightarrow} X$ be a regular embedding of codimension $e$. In other words, the normal cone $C_{Y} X$ of $Y$ in $X$ is in fact a vector bundle of rank $e$. Recall that the normal cone $C_{Y} X$ is the cone over $X$ defined by the sheaf of graded algebras $\bigoplus_{n \geq 0} \mathcal{J}^{n} / \mathcal{J}^{n+1}$, where $\mathcal{J}$ is the ideal sheaf of $Y$ in $X$. In other words, $C_{Y} X=\operatorname{Spec}\left(\bigoplus_{n \geq 0} \mathfrak{g}^{n} / \mathfrak{g}^{n+1}\right)$. When $j: Y \hookrightarrow X$ is a regular embedding, denote $C_{Y} \mathcal{X}$ by $N_{Y} X$. The sheaf of sections of $N_{Y} X$ is $\left(\mathcal{J} / \mathcal{J}^{2}\right)^{*}$.

The main construction is the following. Let $M$ denote the blow-up of $X \times \mathbb{P}^{1}$ along $Y \times\{\infty\}$. The normal bundle to $Y \times \infty$ in $X \times \mathbb{P}^{1}$ is $N \oplus \mathbf{1}$, where $N=N_{Y} X$ and 1 is the trivial line 
bundle. Hence, the exceptional divisor of the blow-up is $\mathbb{P}(N \oplus \mathbf{1})$. Note that $\mathbb{P}(N \oplus \mathbf{1})$ has two "sections" (subvarieties):

$$
\mathbb{P}(N) \equiv \mathbb{P}(N \oplus 0) \hookrightarrow \mathbb{P}(N \oplus \mathbf{1}) \hookleftarrow \mathbb{P}(0 \oplus \mathbf{1}) \equiv Y .
$$

The composition $M \stackrel{b}{\longrightarrow} X \times \mathbb{P}^{1} \underset{P_{1}^{r}}{\longrightarrow} \mathbb{P}^{1}$ gives a flat map $M \stackrel{p}{\longrightarrow} \mathbb{P}^{1}$ satisfying:

(1) The fiber above any $x \neq \infty$ is $X$;

(2) The fiber $p^{-1}(\infty)$ above $\infty$ is a sum of two Cartier divisors: $\rho^{-1}(\infty)=\widetilde{X}+\mathbb{P}(N \oplus \mathbf{1})$, where $\widetilde{X}=\left.B\right|_{Y} X$ is the blow-up of $X$ along $Y$.

Here is a picture of the situation:
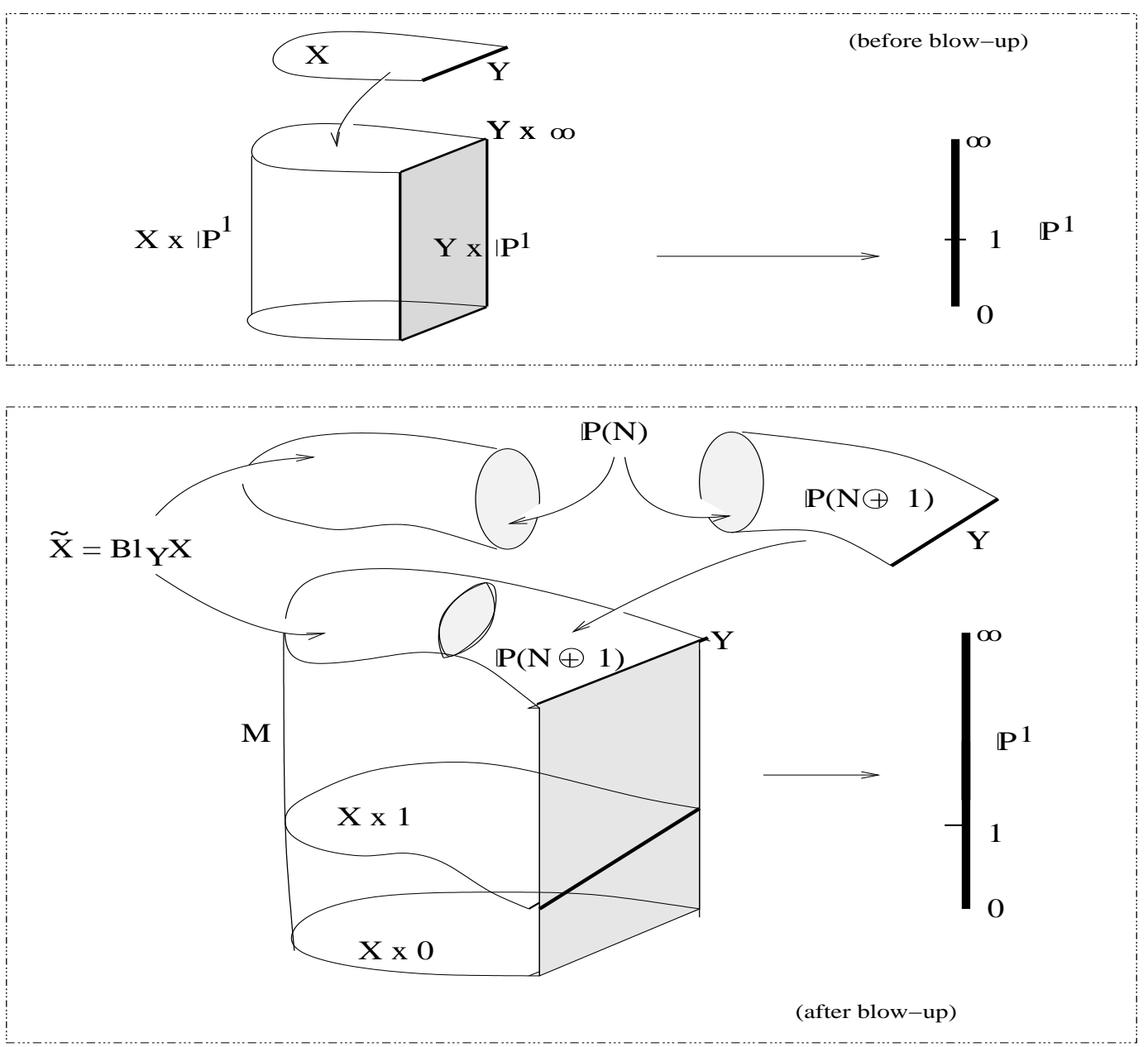
We now introduce the deformation space

$$
M^{0}:=M-\tilde{X},
$$

and note that $p: M^{0} \rightarrow \mathbb{P}^{1}$ is still a flat map whose fiber above $\infty$ is

$$
p^{-1}(\infty)=\mathbb{P}(N \oplus \mathbf{1})-\mathbb{P}(N) \equiv N=N_{Y} X,
$$

and $p^{-1}(t) \equiv X$ for all $t \neq \infty$.

Remark 6.2. A relevant observation is the fact that the normal cone $N_{Y} X$ is a closed subvariety of $M^{0}$ satisfying:

$$
M^{0}-N_{Y} X=X \times \mathbb{A}^{1} .
$$

Remark 6.3. Contrary to the situation in differential geometry, in algebraic geometry one does not have a "tubular neighborhood" of $Y$ in $X$ that can be identified with the normal bundle $N_{Y} X$. In many ways, this deformation space is the best replacement for tubular neighborhoods, allowing one to "localize" information from $X$ to $Y$.

In order to use the ingredients described in the beginning of this section, we start with the following simple observation:

Lemma 6.4. Given an algebraic variety $X$, let $i_{1}: X \hookrightarrow X \times \mathbb{A}^{1}$ denote the inclusion $i_{1}(x)=$ $(x, 1)$ of $X$ as a "slice" $X \times\{1\}, 1 \in \mathbb{A}^{1}$. Then, the multiplication $\mathbb{A}^{1} \times \mathbb{A}^{1} \rightarrow \mathbb{A}^{1}$ sending $(t, s)$ to ts induces a natural homotopy:

$$
\psi_{X}: z_{p}(X) \times I \longrightarrow z_{p}\left(X \times \mathbb{A}^{1}\right)
$$

such that

$$
\begin{aligned}
& \psi_{X}(\sigma, 0)=i_{1 *}(\sigma) \\
& \psi_{X}(\sigma, 1)=0 .
\end{aligned}
$$

In other words, the inclusion $i_{1 *}$ is naturally homotopic to zero.

Proof. Let $\gamma:[0,1] \rightarrow \mathbb{P}^{1}(\mathbb{C})$ denote any path joining $1 \in \mathbb{A}^{1} \subset \mathbb{P}^{1}$ to $\infty \in \mathbb{P}^{1}$.

Consider the composition:

$$
\begin{aligned}
\psi_{X}: z_{p}(X) \times I \longrightarrow z_{p}\left(X \times \mathbb{P}^{1}\right) \longrightarrow{ }_{q} & z_{p}\left(X \times \mathbb{A}^{1}\right) \equiv z_{p}\left(X \times \mathbb{P}^{1}\right) / z_{p}\left(X \times \mathbb{A}^{1}\right) \\
\left(\sigma_{1}, t\right) & \longmapsto i_{\gamma(t)^{*}}(\sigma)
\end{aligned}
$$

where the continuity of $(\sigma, t) \mapsto i_{\gamma(t)^{*}}(\sigma) \equiv \sigma \times \gamma(t)$ is given by Claim 3.7, and $q$ is the quotient map.

Exercise 6.1. Verify that $\psi_{X}$ is the correct homotopy. 
The whole construction can now be summarized in the following diagram:

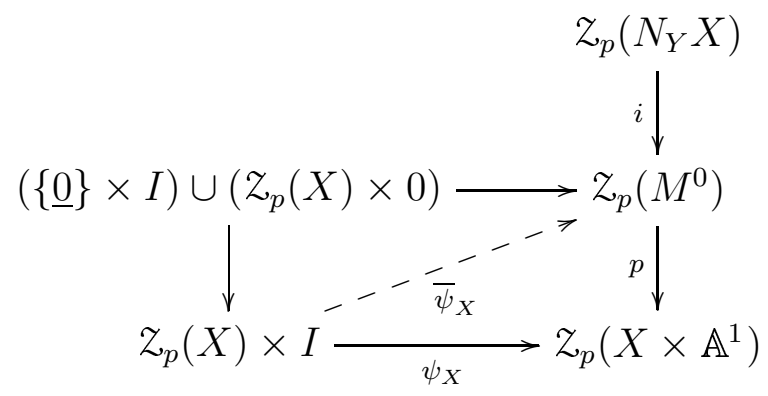

It follows that the map $p$ is a locally trivial fibration with fiber $z_{p}\left(N_{Y} X\right)$, according to Remark 6.2 and Property 4 of Section 2 . Also, Property 5 gives a cofibration $\{\underline{0}\}=Z_{p}(X)_{\leq 0} \hookrightarrow Z_{p}(X)$, i.e. $\underline{0} \in Z_{p}(X)$ is a non-degenerate base point. Hence, we have assembled in the diagram above a classical homotopy-lifting problem, whose solution $\bar{\psi}_{X}: z_{p}(X) \times I \rightarrow z_{p}\left(M^{0}\right)$ satisfies:

(1) $p \circ \bar{\psi}_{X}=\psi_{X}$

(2) $\bar{\psi}(\underline{0}, t)=\underline{0} \quad \forall t$

(3) $\bar{\psi}(\sigma, 0)=i_{1^{*}}(\sigma)$

Since $p \circ \bar{\psi}_{X}(\sigma, 1)=\psi_{X}(\sigma, 1)=\underline{0}$, then $\bar{\psi}_{X}(\sigma, 1) \in p^{-1}(0)=z_{p}\left(N_{Y} X\right) \hookrightarrow z_{p}(X)$. This gives the following:

Definition 6.5. The specialization map

$$
\tau: z_{p}(X) \longrightarrow z_{p}\left(N_{Y} X\right)
$$

is defined as $\tau(\sigma)=\bar{\psi}(\sigma, 1)$.

Remark 6.6. One can show that $\sigma$ is unique up to homotopy. Observe that, up to now, one does not need the regularity of the embedding $Y \underset{j}{\hookrightarrow} X$. Hence, all constructions could have been made for any embedding, with the normal cone $C_{Y} X$ instead of $N_{Y} X$.

At this point, assume that $j: Y \hookrightarrow X$ is a regular embedding, i.e. $N_{Y} X \underset{\pi}{\longrightarrow} Y$ is a vector bundle of rank $e$. Hence, $\pi^{*}: z_{p-e}(Y) \rightarrow z_{p}\left(N_{X} Y\right)$ is a homotopy equivalence, by the homotopy property.

Definition 6.7. Define the Gysin map

$$
j^{!}: z_{p}(X) \longrightarrow z_{p-e}(Y)
$$

as the unique homotopy class of maps: $j^{!}=\left(\pi^{*}\right)^{-1} \circ \bar{h}$, where $\left(\pi^{*}\right)^{-1}$ is the homotopy inverse of $\pi^{*}$, and $\tau$ is the specialization map defined above.

The following properties hold. See [FG93]. 
Theorem 6.8. Let $j: Y \hookrightarrow X$ be a regular embedding of codimension e. The Gysin map $j^{!}: Z_{p}(X) \rightarrow z_{p-e}(Y)$ satisfies:

(1) If $Z_{p}(X)_{\cap Y}^{0}$ is the subgroup of $Z_{p}(X)$ consisting of those cycles intersecting $Y$ properly, then the restriction of $j !$ to $Z_{p}(X)_{\cap Y}^{0}$ is homotopic to the usual intersection theoretic map: $\sigma \mapsto \sigma \cdot Y$.

(2) If $W \stackrel{i}{\longrightarrow} Y$ is another embedding of codimension d, then one has a homotopy:

$$
(j \circ i)^{!} \simeq i^{!} \circ j^{!}: z_{p}(X) \rightarrow z_{p-d-e}(Y) .
$$

(3) Suppose $g: X^{\prime} \rightarrow X$ is flat of relative dimension $r$ and form the pull-back diagram

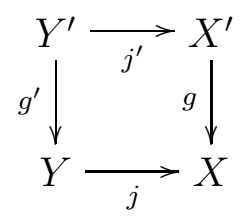

Then one has a homotopy $j^{\prime !} \circ g^{*} \simeq g^{*} \circ j^{!}$. Similarly, if $g$ is proper then $j^{!} \circ g_{*} \simeq g_{*}^{\prime} \circ j^{\prime !}$.

\subsection{Intersection Product in Lawson Homology.}

Using the Gysin maps above, one can prove intersection-theoretic properties of Lawson homology. The same formalism would prove similar properties for Higher Chow groups; cf. Remark 6.1 .

Proposition 6.9. Let $f: X \rightarrow Y$ be a morphism between smooth varieties, where $Y$ is equidimensional, $\operatorname{dim} Y=e$. The graph $\Gamma_{f}$ induces a pairing:

$$
\Gamma_{f}^{!}: L_{p} H_{n}(X) \otimes L_{q} H_{m}(Y) \longrightarrow L_{p+q-e} H_{m+n-2 e}(X) .
$$

Proof. The map $(1 \times f): X \rightarrow X \times Y$ is a regular embedding of codimension $=\operatorname{dim} Y=e$. Consider the composition $z_{p}(X) \times z_{q}(Y) \stackrel{\mu}{\rightarrow} z_{p+q}(X \times Y) \stackrel{(1 \times f)^{!}}{\longrightarrow} z_{p+q-e}(X)$, where $(1 \times f)^{!}$ is the Gysin map from Definition 6.7 and $\mu$ denotes the multiplication of cycles; see Claim 3.7. This composition satisfies $(1 \times f)^{!} \circ \mu(\sigma, \underline{0})=(1 \times f) ! \circ \mu(\underline{0}, \tau)=\underline{0}$, and hence it factors through $z_{p}(X) \wedge z_{q}(Y)$

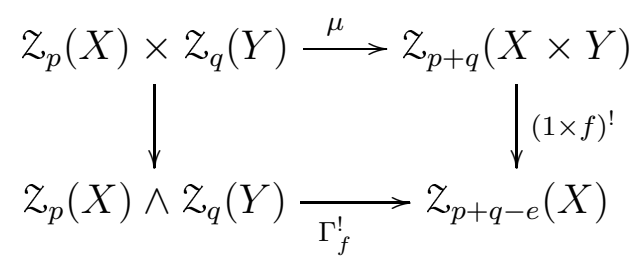

In the level of homotopy groups one obtains a pairing:

$$
\pi_{n-2 p}\left(Z_{p}(X)\right) \otimes \pi_{m-2 q}\left(Z_{q}(Y)\right) \underset{\Gamma_{f}^{!}}{\longrightarrow} \pi_{n+m-2(p+q)}\left(z_{p+q-e}(X)\right),
$$


giving (17).

As a particular case, consider the identity map id: $X \rightarrow X$, hence $\Gamma_{\text {id }}=\Delta: X \rightarrow X \times X$ is the diagonal map. It follows that $\Delta$ induces an intersection pairing:

$$
\Delta^{!}: L_{p} H_{n}(X) \otimes L_{q} H_{m}(X) \rightarrow L_{p+q-e} H_{m+n-2 e}(X),
$$

where $e=\operatorname{dim} X$ and $p+q \geq e$.

The next result follows is a direct consequence of the constructions above and Theorem 6.8.

Theorem 6.10. If $X$ is a smooth variety of dimension $e$ and $p+q \geq e$, then the intersection pairing (18) satisfies:

(1) It is associative and commutative;

(2) In the level of $\pi_{0}$ it induces the usual intersection pairing:

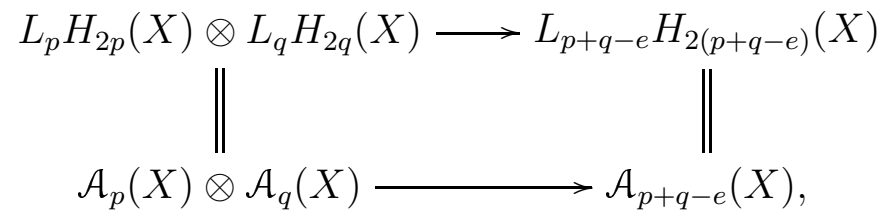

where $\mathcal{A}_{p}(X):=z_{p}(X) / \mathcal{Z}_{p}(X) \mathrm{alg}$.

\section{Morphic COHOMOlOGY}

\subsection{Basic Properties.}

Morphic cohomology is the cohomological counterpart of Lawson homology. It was introduced by Friedlander and Lawson in [FL92] [FL92].

In order to motivate its definition, we first present basic computations. Throughout this section, we switch the notation of cycle spaces and use upperscripts to denote the codimension of cycles. The homotopy property gives homotopy equivalences:

$$
Z^{q}\left(\mathbb{A}^{n}\right) \stackrel{\simeq}{\longleftarrow} Z^{q}\left(\mathbb{A}^{n-1}\right) \stackrel{\simeq}{\longleftarrow} \ldots Z^{q}\left(\mathbb{A}^{q}\right)
$$

Hence

$$
\begin{aligned}
\pi_{k}\left(Z^{q}\left(\mathbb{A}^{n}\right)\right) & \cong \pi_{k}\left(Z^{q}\left(\mathbb{A}^{q}\right)\right)=\pi_{k}\left(Z_{0}\left(\mathbb{A}^{q}\right)\right) \\
& \cong H_{k}^{\mathrm{BM}}\left(\mathbb{A}^{q} ; \mathbb{Z}\right) \cong \begin{cases}0 & ; k \neq 2 q \\
\mathbb{Z} ; & k=2 q\end{cases}
\end{aligned}
$$

This shows that $Z^{q}\left(\mathbb{A}^{n}\right)$ is an Eilenberg-MacLane space of type $K(\mathbb{Z}, 2 q)$. In particular, $[X, K(\mathbb{Z}, 2 q)] \cong$ $H^{2 q}(X ; \mathbb{Z})$ for every finite $C W$-complex $X$. 
Remark 7.1. If one keeps track of Hodge structures, $\pi_{2 q}\left(Z_{0}\left(\mathbb{A}^{q}\right)\right)$ with its Hodge structure, induced from the Borel-Moore homology of $\mathbb{A}^{q}$, is in fact $\mathbb{Z}(q)$. Hence $Z^{q}\left(\mathbb{A}^{n}\right)$ is better identified with $K(\mathbb{Z}(q), 2 q)$ when $n \geq q$. Furthermore, iterations of the cycle map give

$$
\pi_{2 q}\left(Z^{q}\left(\mathbb{A}^{n}\right)\right)=L_{n-q} H_{2 n}\left(\mathbb{A}^{n}\right) \stackrel{s^{n-q}}{\longrightarrow} L_{0} H_{2 n}\left(\mathbb{A}^{n}\right) \otimes \mathbb{Z}(q-n) \cong \mathbb{Z}(n) \otimes \mathbb{Z}(q-n) \cong \mathbb{Z}(q),
$$

and this identification of pure Hodge structures holds for every $n \geq q$.

For simplicity we assume that all varieties are weakly normal, and the term "Chow varieties" refers to the weak normalization of the usual Chow varieties.

Definition 7.2. [FL92] An effective algebraic s-cocycle on a variety $X$ with values in a projective variety $Y$ is a morphism $\varphi: X \longrightarrow \mathrm{e}^{s}(Y)$, where $\mathrm{e}^{s}(Y)$ is the Chow monoid of cycles of codimensions in $Y$. Denote by $\mathrm{e}^{s}(X ; Y)$ the set of all such $s$-cocycles.

\section{Examples 7.3.}

a. Any cycle $\Gamma \in \mathfrak{e}^{s}(X \times Y)$ which is flat over $X$ gives an $s$-cocycle $\pi_{\Gamma}: X \rightarrow \mathcal{e}^{s}(Y)$; see Section 2.

b. $X \subset \mathbb{P}^{N}$ smooth hypersurface and $Y \subset \mathbb{P}^{N}$ is projective subvariety. Define

$$
\varphi: X \rightarrow \mathcal{C}^{1}(Y)
$$

by $\varphi(x)=\left(T_{p} X\right) \cdot Y$, where the latter denotes the intersection-theoretic product.

Construction 7.4. Any effective cocycle $\varphi \in \mathcal{C}^{s}(X ; Y)$ can be transformed, via a "graphing construction", into a cycle $\Gamma_{\varphi} \in \mathrm{e}^{s}(X \times Y)$ which is equidimensional over $X$; cf. [FL92].

Up to appropriate modifications in the resulting topology, we can think of the set $\mathrm{e}^{s}(X ; Y)$ as a subspace of Map $\left(X(\mathbb{C}) ; \mathfrak{C}^{s}(Y)\right)$, with the compact-open topology.

Theorem 7.5. [FL92] If $X$ is projective and irreducible, the graphing map

$$
\Gamma: \mathfrak{e}^{s}(X ; Y) \longrightarrow \mathfrak{e}^{s}(Y / X)^{e q}
$$

is a homeomorphism. Here, $\mathrm{e}^{s}(Y / X)^{e q}$ denotes the submonoid of the Chow monoid $\mathrm{e}^{s}(Y \times X)$ consisting of cycles that are equidimensional over $X$.)

This theorem allows one to have a good grasp on the topology of $\mathcal{e}^{s}(X ; Y)$.

Definition 7.6. Define the morphic cohomology of $X$ with values in $Y$ by

$$
L^{q} H^{n}(X ; Y)=\pi_{2 q-n}\left(Z^{q}(X ; Y)\right),
$$

where $Z^{q}(X, Y)$ denotes the group completion $Z^{q}(X ; Y):=\Omega B\left(\mathrm{e}^{q}(X ; Y)\right)$ of the monoid $\mathrm{e}^{q}(X ; Y)$. 
Returning to the motivation given at the beginning of this section, let $T$ be a compact $C W$ complex, and let $K(\mathbb{Z}(q), 2 q)$ be an Eilenberg-MacLane space. Then one has canonical isomorphisms:

$$
\begin{aligned}
\pi_{2 q-n}\left(\operatorname{Map}_{\bullet}(T, K(\mathbb{Z}(q), 2 q))\right) & \cong\left[S^{2 q-n} ; \operatorname{Map}(T, K(\mathbb{Z}(q) ; 2 q))\right] \\
& =\left[S^{2 q-n} \wedge T ; K(\mathbb{Z}(q), 2 q)\right] \cong\left[T ; \Omega^{2 q-n} K(\mathbb{Z}(q) ; 2 q)\right] \\
& \cong[T ; K(\mathbb{Z}(q), n)]=H^{n}(X ; \mathbb{Z}(q)) .
\end{aligned}
$$

In morphic cohomology, we replace Map by Mor, and use $Z^{q}\left(\mathbb{A}^{n}\right)$ as an "algebraic geometric" model for $K(\mathbb{Z}(q), 2 q)$.

Definition 7.7. For $n \geq q$, denote

$$
Z^{q}\left(X ; \mathbb{A}^{n}\right):=Z^{q}\left(X ; \mathbb{P}^{n}\right) / Z^{q-1}\left(X ; \mathbb{P}^{n-1}\right)
$$

(homotopy quotient) and define the morphic cohomology groups of $X$ by

$$
L^{q} H^{n}(X):=\pi_{2 q-n}\left(Z^{q}\left(X ; \mathbb{A}^{n}\right)\right)
$$

An application of Lawson's suspension theorem (modified arguments for families), gives canonical homotopy equivalences

$$
Z^{q}\left(X ; \mathbb{A}^{n}\right) \simeq Z^{q}\left(X ; \mathbb{A}^{n+1}\right) \simeq \cdots
$$

hence, the definition of morphic cohomology does not depend on $n$.

Properties 7.8. (1) Morphic cohomology is contravariantly functorial for arbitrary morphisms.

(2) If $f: X \rightarrow X^{\prime}$ is proper and flat of relative dimension e, there are Gysin maps:

$$
f_{!}: L^{q} H^{n}(X) \longrightarrow L^{q+e} H^{n+2 e}\left(X^{\prime}\right) .
$$

$(f ! \circ g !=(f \circ g) !$ for two such maps $)$.

(3) The join pairing induces a ring structure on $L^{\bullet} H^{\bullet}$. I.e. the pointwise multiplication

$$
\left(\varphi \# \varphi^{\prime}\right)(x)=\varphi(x) \# \varphi\left(x^{\prime}\right)
$$

for elements $\varphi \in \mathcal{C}^{q}\left(X ; \mathbb{P}^{n}\right), \varphi^{\prime} \in \mathfrak{C}^{q^{\prime}}\left(X ; \mathbb{P}^{n^{\prime}}\right)$, induces a pairing

$$
L^{q} H^{r}(X) \otimes L^{q^{\prime}} H^{r^{\prime}}(X) \rightarrow L^{q+q^{\prime}} H^{r+r^{\prime}}(X)
$$

once one descends to homotopy quotients and takes homotopy groups. This makes $L^{\bullet} H^{\bullet}(X)$ a bigraded commutative ring.

(4) Cycle Maps: The forgetful functor from Mor to Map induces a map:

$$
Z^{q}\left(X ; \mathbb{A}^{n}\right) \rightarrow \operatorname{Map}\left(X(\mathbb{C}) ; Z^{q}\left(\mathbb{A}^{n}\right)\right)
$$


In the level of homotopy groups, this gives natural homomorphisms

$$
\begin{aligned}
L^{q} H^{n}(X) & :=\pi_{2 q-n}\left(Z^{q}\left(X ; \mathbb{A}^{n}\right)\right) \rightarrow \pi_{2 q-n}\left(\operatorname{Map}\left(X(\mathbb{C}) ; Z^{q}\left(\mathbb{A}^{n}\right)\right)\right) \\
& \cong\left[X(\mathbb{C}) ; \Omega^{2 q-n} Z^{q}\left(\mathbb{A}^{n}\right)\right] \cong[X(\mathbb{C}) ; K(\mathbb{Z}(q) ; n)] \\
& \cong H^{n}(X(\mathbb{C}) ; \mathbb{Z}(q))
\end{aligned}
$$

cf. Remark 7.1 and (19). Hence one gets cycle maps:

$$
\Phi: L^{p} H^{n}(X) \longrightarrow H^{n}(X ; \mathbb{Z}(p)) .
$$

Example 7.9. It is shown in [FL92] that in the case of divisors one has the following:

Theorem. For any projective variety $X$ there is a natural homotopy equivalence:

$$
Z^{1}(X) \cong \operatorname{Pic}(X) \times \mathbb{P}^{\infty}
$$

If $X$ is smooth, then

1) $L^{1} H^{0}(X) \cong \mathbb{Z}$

2) $L^{1} H^{1}(X) \cong H^{1}(X ; \mathbb{Z})$

3) $L^{1} H^{2}(X) \cong N S(X)$

4) The cycle map:

$$
\Phi: L^{1} H^{2}(X) \cong N S(X) \longrightarrow H^{2}(X ; \mathbb{Z}(1))
$$

is the first Chern class map.

5) $L^{1} H^{k}(X)=0$ for $k>2$.

\subsection{Duality.}

There is a very simple duality map between Lawson homology and morphic cohomology. Recall that one has an inclusion $\mathcal{\complement}^{q}\left(X ; \mathbb{P}^{n}\right) \hookrightarrow \mathcal{C}^{q}\left(X \times \mathbb{P}^{n}\right)$, hence one can pass to group completions and homotopy quotients to get a map

$$
Z^{q}\left(X ; \mathbb{A}^{n}\right) \longrightarrow Z^{q}\left(X \times \mathbb{A}^{n}\right)=z_{d-q+n}\left(X \times \mathbb{A}^{n}\right),
$$

where $d=\operatorname{dim} X$. Using the homotopy property one obtains a (homotopy class) map:

$$
Z^{q}\left(X ; \mathbb{A}^{n}\right) \longrightarrow Z_{d-q}(X)
$$

which, when one applies the homotopy group functor $\pi_{2 q-r}$ gives:

$$
\mathcal{D}: L^{q} H^{r}(X) \longrightarrow L_{d-q} H_{2 d-r}(X) \text {. }
$$

This is the "duality homomorphism". 
Theorem. [FL97] For any projective variety of dimension d, one gets a commutative diagram:

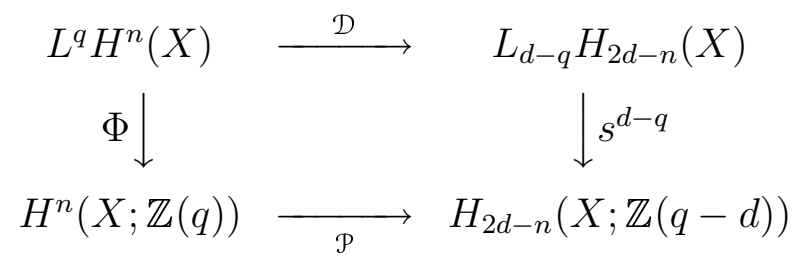

where $\mathcal{P}$ is the Poincaré duality map given by cap product with the fundamental class $[X] \in$ $H_{2 d}(X, \mathbb{Z}(d))$ of $X$.

In fact, as a beautiful consequence of their moving lemma for families [FL98], Friedlander and Lawson prove that one has duality isomorphisms:

Theorem 7.10. [FL97] For a smooth projective variety $X$, of dimension $d$, the duality map:

$$
\mathcal{D}: L^{q} H^{n}(X) \longrightarrow L_{d-q} H_{2 d-n}(X)
$$

is an isomorphism.

\section{REFERENCES}

[Alm62] F. J. Almgren, Jr., Homotopy groups of the integral cycle groups, Topology 1 (1962), 257-299.

[Blo86] S. Bloch, Algebraic cycles and higher K-theory, Adv. in Math. 61 (1986), 267-304.

[BO84] S. Bloch and A. Ogus, Gersten's conjecture and the homology of schemes, Ann. Sci. École Norm. Sup. (4) 7 (1984), 181-202.

[Del71] P. Deligne, Théorie de Hodge II, Inst. Hautes Études Sci. Publ. Math. 40 (1971), 5-58.

[Del74] _ Théorie de Hodge III, Inst. Hautes Études Sci. Publ. Math. 44 (1974), 5-77.

[dS] P. dos Santos, Algebraic cycles on real varieties and $\mathbb{Z} / 2$-homotopy theory, Preprint (2000), http://www. dossantos. com/paper.pdf.

[dSLF] P. dos Santos and P. Lima-Filho, Quaternionic algebraic cycles and reality, Preprint (2001).

[DT56] A. Dold and R. Thom, Quasifaserungen und unendlich symmetrische Produkte, Ann. of Math. 67 (1956), no. 2, 230-281.

[Fed69] H. Federer, Geometric measure theory, 1st ed., Springer-Verlag, New York, 1969.

[FG83] W. Fulton and H. Gillet, Riemann-Roch for general algebraic varieties, Bull. Soc. Math. France 111 (1983), 287-300.

[FG93] E. M. Friedlander and O. Gabber, Cycle spaces and intersection theory, Topological Methods in Modern Mathematics (Austin, TX), Publish or Perish, 1993, Conference in Honor to J. Milnor, pp. 325-370.

[FL92] E. M. Friedlander and H. B. Lawson, Jr., A theory of algebraic cocycles, Ann. of Math. (2) 136 (1992), 361-428.

[FL97] _ Duality relating spaces of algebraic cocycles and cycles, Topology 36 (1997), no. 2, 533-565.

[FL98] _ Moving algebraic cycles of bounded degree, Invent. Math. 132 (1998), 91-119.

[FM94] E. M. Friedlander and B. Mazur, Filtrations on the homology of algebraic varieties, vol. 110, Mem. Amer. Math. Soc., no. 529, AMS, 1994.

[Fri91] E. M. Friedlander, Algebraic cycles, Chow varieties and Lawson homology, Compositio Math. 77 (1991), 55-93. 
[Fri95] _ Filtrations on algebraic cycles and homology, Ann. Sci. École Norm. Sup. (4) 28 (1995), no. 4, 317-343.

[Ful84] W. Fulton, Intersection theory, 1st ed., Springer-Verlag, Heidelberg, 1984.

[Gri68] P. Griffiths, Periods of integrals on algebraic manifolds, Amer. J. of Math. 90 (1978), 568-626.

[GH78] P. Griffiths and J. Harris, Principles of Algebraic Geometry, John Wiley \& Sons, New York, 1978.

[GD66] A. Grothendieck and J. Dieudonné, Elements de geometrie algebrique IV - part 3, Inst. Hautes Études Sci. Publ. Math. 28 (1966), 5-255.

[Hir75] H. Hironaka, Triangulation of algebraic sets, Proc. of Symp. in Pure Math. 29 (1975), 165-185.

[Hod41] W. V. D. Hodge, The theory and applications of harmonic integrals, Cambridge University Press, 1941.

[Kin83] J. R. King, Log complexes of currents and functorial properties of the Abel-Jacobi map, Duke Math. J. 50 (1983), 1-53.

[Ko196] J. Kollár, Rational curves on algebraic varieties, Ergebnisse der Mathematik und ihrer Grenzgebiete, vol. 32, Sprin-Verlag, Berlin, 1996.

[Law89] H. B. Lawson, Jr., Algebraic cycles and homotopy theory, Ann. of Math. (2) 129 (1989), $253-291$.

[Law95] _ Spaces of algebraic cycles, Surveys in Differential Geometry, vol. 2, pp. 137-213, International Press, 1995.

[LF92] P. Lima-Filho, Lawson homology for quasiprojective varieties, Compositio Math. 84 (1992), 1-23.

[LF93a] _ Completions and fibrations for topological monoids, Trans. Amer. Math. Soc. 340 (1993), 127147.

[LF93b] _ On the generalized cycle map, J. Differential Geom. 38 (1993), no. 1, 105-129.

[LF94] _ The topological group structure of algebraic cycles, Duke Math. J.. 75 (1994), no. 2, 467-491.

[LLFM] H. B. Lawson, Jr., P. Lima-Filho, and M.-L. Michelsohn, Algebraic cycles and the classical groups, I; Real Cycles, To appear in TOPOLOGY.

[LLFM96] __ Algebraic cycles and equivariant cohomology theories, Proc. London Math. Soc. (3) 73 (1996), no. $3,679-720$.

[LLFM98] _ On equivariant algebraic suspension, J. Algebraic Geometry 7 (1998), no. 4, 627-650.

[LM88] H. B. Lawson, Jr. and M.-L. Michelsohn, Algebraic cycles, Bott periodicity, and the Chern characteristic map, Proc. of Symp. in Pure Math. 48 (1988), 241-264.

[May75] J. P. May, Classifying spaces and fibrations, Mem. Amer. Math. Soc., vol. 155, AMS, Providence, RI, 1975.

[May82] _ Simplicial objects in algebraic topology, Midway Reprint, Univ. of Chicago Press, Chicago, IL, 1982.

[MV99] F. Morel and V. Voevodsky, $\mathbb{A}^{1}$-homotopy theory of schemes, Inst. Hautes Études Sci. Publ. Math. 90 (1999), 45-143.

[Nor93] M. V. Nori, Algebraic cycles and hodge theoretic connectivity, Inventiones Math. 111 (1993), no. 2, 349-373.

[Roj71] A. A. Rojtman, On $\gamma$-equivalence of zero-dimensional cycles, Math. USSR Sbornik 15 (1971), 555-567.

[Roj72] _ Rational equivalence of zero-cycles, Math. USSR Sbornik 18 (1972), 571-588.

[Seg74] G. Segal, Categories and cohomology theories, Topology 13 (1974), 193-312.

[Ste67] N. Steenrod, A convenient category of topological spaces, Michigan Math. J. 14 (1967), $133-152$. 
Department of Mathematics, Texas A\&M University, College Station, Texas 77840

E-mail address, Lima-Filho: plfilho@math.tamu.edu 